Article

\title{
The Entropic Dynamics of Quantum Scalar Fields Coupled to Gravity
}

\author{
Selman Ipek * and Ariel Caticha \\ Physics Department, University at Albany-SUNY, Albany, NY 12222, USA; acaticha@albany.edu \\ * Correspondence: sipek@albany.edu
}

Received: 9 June 2020; Accepted: 9 July 2020; Published: 7 August 2020

\begin{abstract}
Entropic dynamics (ED) are a general framework for constructing indeterministic dynamical models based on entropic methods. ED have been used to derive or reconstruct both non-relativistic quantum mechanics and quantum field theory in curved space-time. Here we propose a model for a quantum scalar field propagating in dynamical space-time. The approach rests on a few key ingredients: (1) Rather than modelling the dynamics of the fields, ED models the dynamics of their probabilities. (2) In accordance with the standard entropic methods of inference, the dynamics are dictated by information encoded in constraints. (3) The choice of the physically relevant constraints is dictated by principles of symmetry and invariance. The first of such principle imposes the preservation of a symplectic structure which leads to a Hamiltonian formalism with its attendant Poisson brackets and action principle. The second symmetry principle is foliation invariance, which, following earlier work by Hojman, Kuchař, and Teitelboim, is implemented as a requirement of path independence. The result is a hybrid ED model that approaches quantum field theory in one limit and classical general relativity in another, but is not fully described by either. A particularly significant prediction of this ED model is that the coupling of quantum fields to gravity implies violations of the quantum superposition principle.
\end{abstract}

Keywords: quantum gravity; general covariance

\section{Introduction}

Without any empirical matter of fact, and none likely on the near horizon, quantum gravity (QG) research has largely split off into distinct channels, each reflecting a different set of attitudes, and yes, philosophies directed towards the problem at hand. (See e.g., [1] for a recent overview of some feasible experimental proposals). But this state of affairs should not be entirely surprising. Solving the problem of QG should, after all, entail first addressing the issue of what QG even is-indeed, what is meant by "quantum"? What is meant by gravity? Which, if any, elements of Einstein's gravity, or the standard quantum formalism, should be abandoned in the transition to QG?

Pursuant to this, a common view is that the gravitational field itself should, in some way, be quantized. There are several routes to accomplishing this. (For other popular alternatives, see e.g., [2,3]). Most typical, however, is through some manner of quantization algorithm: extending the existing quantum formalism to the gravitational domain by application of the standard ad hoc quantization rules to the appropriate gravitational degrees of freedom. Such is the tack taken by the principal QG candidates-string theory (ST) and loop quantum gravity (LQG). (For some historical overviews in the development of ST and LQG, see the reviews by S. Mukhi [4] and C. Rovelli [5], respectively).

But such approaches should be met with some suspicion in the context of QG. One issue is the quantization procedure itself. Take models with constraints, such as general relativity (GR), for instance. Should we solve the constraints and then quantize, or quantize and then solve the constraints? The two methods are not, in general, the same (see, e.g., [6]). Another issue is choosing 
which degrees of freedom to quantize in the first place. Historically, this process has been guided by tight coordination between theory and experiment, allowing trial and error to supplement an incomplete understanding of the quantization process itself. But absent such help from experiments, a more fundamental understanding of quantization may be necessary to make progress.

The lack of clarity around quantization is made particularly acute in the case of the gravitational field, which plays a dual role in GR as an object with genuine dynamical modes, but one that also serves to establish spatial and temporal relationships. This calls into question the precise nature of the gravitational field. Is it just another field to be quantized, along the lines of the gauge fields of Yang-Mills theories, or is it something else entirely? Canonical approaches to QG, such as LQG, have wagered, with varying degrees of success, as to whether the former is true. But there are various clues (see, e.g., [7-11]) suggesting that gravity is an emergent, statistical phenomenon, not unlike temperature and pressure in statistical physics. Would any physicist quantize a temperature field? Perhaps not, but this is exactly what we might be doing when we quantize the gravitational field.

All of this suggests that a different attitude with regard to QG may be in order. In the past couple of decades there has been increased interest (see, e.g., [12-17]) in developing approaches to QG that seek to treat gravitational considerations hand-in-hand with a more robust understanding of quantum theory (QT). The goal in these cases, however, is not just the development of specific models (although such models must necessarily follow), but the construction of entire frameworks that can readily integrate salient features of gravitational and quantum physics alike. One such approach is afforded by entropic dynamics (ED), which is a general framework for constructing indeterministic dynamical models based on the principles of Bayesian probability and entropic inference. (For comprehensive overview of Bayesian and entropic methods, see, e.g., [18,19]).

Among the successes of the ED framework are principled derivations of several aspects of the quantum formalism [20-30], which have led to many key insights. Foremost among these is the notion of probability itself. In ED, probabilities are Bayesian-that is, there are no classical probabilities or quantum probabilities; there are simply probabilities and they follow the usual rules of probability theory [31]. But note that this is not a trivial statement. Taking the structure of probability seriously is, in fact, highly constraining. For instance, once we adopt a probabilistic viewpoint, a natural question that follows is, probabilities of what? Are we dealing with the outcomes of an experimental device, the configurations of fields, or the positions of particles? There is no flexibility here. We must choose-a notion that stands in stark contrast to the usual Copenhagen interpretation. Equally constraining is how probabilities are updated in ED. Indeed, it is not enough to simply declare that $|\Psi|^{2}$ yields a Bayesian probability-one must also demonstrate that the rules for updating those probabilities are in strict accordance with the Bayesian and entropic methods of inference. In short: ED is a dynamical framework driven by constraints.

One of the essential challenges in ED is therefore the appropriate identification of constraints. There have been a series of developments in this regard by appealing to principles of symmetry and invariance, which have rich traditions in both physics [32] and inference [33,34] alike. Early progress in ED [20,21], aided by insights from Nelson's stochastic mechanics [35], was made by recognizing that conservation of a suitable energy functional could be used as the main criterion for updating the evolving constraints. This allowed ED to model many important aspects of quantum mechanics, leading eventually to a fully Hamiltonian formalism, with its attendant action principle, a symplectic structure, and Poisson brackets [22]. However, a sharper understanding of the deep role played by isometries and symplectic symmetries in QT (see, e.g., [36-40]) suggested another path wherein symplectic and metric structures take a more fundamental place in the ED approach [23]. Issues, such as the single-valued nature of the wave function $\Psi$, or more importantly, the linearity of quantum time evolution, are clarified from this perspective as resulting from the marriage of symmetry principles with the probabilistic structure of ED. (For issues related to single-valuedness in QT, see e.g., [41]. See [23] for how this is resolved in ED). 
Building on these developments are the previous efforts by Ipek, Abedi, and Caticha (IAC) [28,29] to model the ED of quantum scalar fields on a fixed curved background. Of particular interest to us are the manifestly covariant methods developed by IAC, which introduced several novel features to ED. One such contribution pertains to the role of time. In ED, time, or better yet, entropic time is constructed as a scheme to keep track of the accumulation of small changes [21]. In previous work, in the context of a flat space-time [26,27], it was appropriate to introduce a global notion of time, in which all spatial points were updated uniformly. As discussed in $[28,29]$, however, this assumption must be relaxed in a curved space-time in favor of a local notion of entropic time. This raises an important challenge, namely, the construction of an updating scheme that is local in nature.

To this end, the work done by IAC in $[28,29]$ synthesized two developments in ED: (I) the adoption of a symplectic structure, together with its accompanying Poisson bracket formalism and (II) an updating scheme that unfolds in local entropic time. The two pieces work in tandem. The former allows ED to marshal the full power of the canonical theory, which, in turn, facilitates the desired local time dynamics. In [28,29], this latter ingredient was itself inspired by the seminal works of Dirac [42,43], and those of Hojman et al. [44], Kuchař [45], and Teitelboim [46,47] (DHKT) in their development of covariant canonical methods in classical field theory. (These approaches could themselves be traced to the earlier "many-time" efforts of Weiss [48], Tomonaga [49], Dirac [50], and Schwinger [51] in the context of relativistic quantum theory).

Drawing on the ideas of DHKT, the IAC framework proceeds as follows. A chief concern is the notion of an instant, which is defined by a three-dimensional space-like surface embedded in space-time; plus the fields, probability distributions, and so on, which are defined on these surfaces. It is then possible to slice or foliate space-time into a sequence of such space-like surfaces. While the decomposition of space-time into spatial slices, indeed, obscures the local Lorentz symmetry of the full pseudo-Riemannian manifold, manifest covariance can be recovered by requiring that the dynamics be unaffected by the particular choice of foliation. This foliation invariance can itself be implemented by a consistency condition dubbed path independence [45,46]: the evolution of all dynamical quantities from an initial to a final surface must be independent of the choice of intermediate surfaces.

The requirement of path independence, as laid out by DHKT, thereby imposes certain conditions on the generators of the local time dynamics. Crucially important, however, is not simply the existence of such conditions, which merely reflects the constraints of path independence, but that these conditions are of a universal nature. Put another way, any attempt to formulate dynamics that unfold in space-time must mirror this pattern, which is itself reflective of the structure of space-time deformations. Thus ED, which is designed as a dynamical scheme that evolves step-by-step from one instant to the next, can be made manifestly covariant by imposing just such a structure. This results in a relativistic ED where quantum fields evolve on a fixed classical background, generalizing the pioneering efforts of DHKT which dealt solely with the evolution of classical fields.

Here we model the indeterministic dynamics of a scalar field $\chi(x)$ interacting with classical gravity. Although there is a growing body of work on modifications of classical GR as a prelude to its eventual quantization, in this paper we pursue a more modest goal: to explore the consequence of a first principles derivation of quantum field theory (QFT) while maintaining classical gravity unmodified. Thus our current work, which expands on [30], extends the efforts of IAC in one crucial respect by allowing the background geometry itself to become dynamical. Following DHKT, the transition to a dynamical background is not accomplished by altering the conditions of path independence, but rather, it is made by an appropriate choice of variables for describing the evolving geometry. The result is a hybrid ED model that approaches QFT in a fixed background in one limit and approaches classical general relativity in another limit, but is not fully described by either. In particular, the model shares some formal similarities with the so-called semi-classical Einstein equations (SCEE) (see, e.g., [52]), but here we model the fluctuations of the quantum fields and derive their coupling to classical gravity from first principles without the ad hoc arguments typically used to justify the SCEE. 
To summarize, in dynamics based on inference, the relevant physical information is supplied through constraints. In the present work the constraints are chosen so as to enforce the symmetry and invariance principles that lie at the foundation of quantum theory and general relativity. More explicitly, we impose that the dynamics be such as to preserve the symplectic structure and to enforce path independence which amounts to foliation invariance and local Lorentz invariance.

An important feature of the ED derivation of nonrelativistic quantum mechanics [23] is its analysis of the superposition principle which led to the recognition that the linearity of quantum mechanics is a consequence of the introduction of Hilbert spaces. After all, this is precisely the reason why Hilbert spaces are introduced in the first place: while in principle they are not needed for the formulation of quantum mechanics, their introduction is nevertheless a very convenient calculational trick because it allows one to make use of the calculational advantages of the linearity they induce. A significant result of our present ED reconstruction of a relativistic QFT coupled to gravity is that the dynamics are fundamentally nonlinear. Not only does this imply violations of the quantum superposition principle, but it brings into question the very reason for Hilbert spaces.

Incidentally, this also makes explicit the fundamental disagreement between the ED approach and other approaches, such as the orthodox Copenhagen and the many-worlds interpretation. (Excellent reviews with extended references to the literature are given in, e.g., [53,54]. The many-worlds interpretation is discussed in [55]). In these interpretations, the linear structure of the Hilbert space, including the superposition principle, is given priority, while the probabilistic structure is either a secondary addition designed to handle those mysterious physical processes called measurements, or, as in the many-worlds interpretation, it is avoided altogether. The unwelcome result is that the dynamical and the probabilistic aspects of quantum theory are essentially incompatible with each other. In contrast, ED resolves these problems by giving priority to the probabilistic structure of QM which relegates Hilbert spaces to play a secondary, non-fundamental role. Indeed, in the particular problem discussed here-quantum fields coupled to dynamical gravity-the dynamics are intrinsically nonlinear; there is no superposition principle, and therefore no reason for Hilbert spaces.

The outline of the paper is the following. In Section 2, we review the ED of short steps. Following this, in Section 3 we introduce some key notation, which is useful in our development of entropic time in Section 4. Key concepts of space-time deformations and "embeddability" are introduced in Section 5. In Section 6 we introduce the canonical formalism in ED, which is a necessary step before we review the condition of path independence developed by DHKT in Section 7. Section 8 outlines the construction of the local generators. In Section 9 we describe the resulting dynamical equations, and in Section 10 we apply these results to obtain an ostensibly quantum theory. We discuss our results in Section 11.

\section{Statistical Model for Short Steps}

We present a short review of the ED of infinitesimal steps in curved space-time, adopting the notations and conventions of [28-30]. Here the object of analysis is a single scalar field $\chi(x) \equiv \chi_{x}$ that populates space and whose values are posited to be definite, but unknown, and thus amenable to being described by probabilities. An entire field configuration, which we denote $\chi$, lives on a 3-dimensional space $\sigma$, the points of which are labeled by coordinates $x^{i}(i=1,2,3)$. The space $\sigma$ is itself curved and comes equipped with a metric $g_{i j}$ that is currently fixed, but that will later become dynamical. A single field configuration $\chi$ is a point in an $\infty$-dimensional configuration space $\mathcal{C}$. Our uncertainty in the values of this field is therefore quantified by a probability distribution $\rho[\chi]$ over $\mathcal{C}$, so that the probability that the field attains a value $\hat{\chi}$ in an infinitesimal region of $\mathcal{C}$ is $\operatorname{Prob}[\chi<\hat{\chi}<\chi+\delta \chi]=\rho[\chi] D \chi$, where $D \chi$ is an integration measure over $\mathcal{C}$.

\subsection{On Microstates}

A virtue of the ED approach is that it achieves ontological clarity by insisting on a sharp distinction between its ontic and epistemic elements. A concept is "ontic" when it describes something real that exists independently of any observer. In the words of John Bell, ontic variables are beables. A concept is 
"epistemic" when it is related to the state of knowledge, opinion, or belief of an agent, albeit an ideally rational agent. Examples of epistemic quantities are probabilities, entropies, and wave functions. An important point is that the distinctions ontic/epistemic and objective/subjective are not the same. Probabilities are fully epistemic — they are tools for reasoning with incomplete information — but they can lie anywhere in the spectrum from being completely subjective (two different agents can have different beliefs) to being completely objective. In QM, for example, probabilities are epistemic and objective-there is such a thing as an objectively correct assignment of probabilities. We will say that the wave function $\Psi$, which is fully epistemic and objective, represents a "physical" state when it represents information about an actual "physical" situation.

In ED the field distributions $\chi_{x}$ play a singularly special role: they define the ontic state of the system. This ontological commitment is in direct contrast with the usual Copenhagen interpretation in which such microscopic values become actualized only through the process of measurement. The Bohmian interpretation shares with ED that fact that the fields are ontic but the resemblance ends there; Bohmian wave functions are ontic, while ED wave functions are fully epistemic $[23,56]$. The metric $g_{i j}$, on the other hand, in our approach is a tool whose purpose is to measure distances, areas, etc., and to characterize the spatial relations between the physical degrees of freedom, the $\chi_{x}$. While the geometry may later become dynamical, we do not interpret this to mean that $g_{i j}$ is itself an ontic variable; it is not. (A model in which the metric tensor is itself of statistical origin is proposed in [10]). Put another way, the geometric variables enter much like parameters in a typical statistical model. The values of those parameters are important in guiding the distribution of outcomes. However, unlike the ontic variables, their values are not detected directly, but inferred from an ensemble of measurements.

\subsection{Maximum Entropy}

Our goal is to predict the indeterministic dynamics of the scalar field $\chi$ whose statistical features are captured by a probabilistic model. To this end, we make one major assumption: in ED, the fields follow continuous trajectories such that finite changes can be analyzed as an accumulation of many infinitesimally small ones. Such an assumption allows us to focus our interest on obtaining the probability $P\left[\chi^{\prime} \mid \chi\right]$ of a transition from an initial configuration $\chi$ to a neighboring $\chi^{\prime}=\chi+\Delta \chi$. This is accomplished via the maximum entropy (ME) method (for an accessible introduction, see, e.g., [18]) by maximizing the entropy functional

$$
S[P, Q]=-\int D \chi^{\prime} P\left[\chi^{\prime} \mid \chi\right] \log \frac{P\left[\chi^{\prime} \mid \chi\right]}{Q\left[\chi^{\prime} \mid \chi\right]^{\prime}}
$$

relative to a prior $Q\left[\chi^{\prime} \mid \chi\right]$ and subject to appropriate constraints.

\subsubsection{The Prior}

We adopt a prior $Q\left[\chi^{\prime} \mid \chi\right]$ that incorporates the information that the fields change by infinitesimally small amounts, but is otherwise maximally uninformative. In particular, before the constraints are taken into account, knowledge of the dynamics at one point does not convey information about the dynamics at another point; i.e., the degrees of freedom are a priori uncorrelated.

Such a prior can itself be derived from the principle of maximum entropy. Indeed, maximize

$$
S[Q, \mu]=-\int D \chi^{\prime} Q\left[\chi^{\prime} \mid \chi\right] \log \frac{Q\left[\chi^{\prime} \mid \chi\right]}{\mu\left[\chi^{\prime}\right]}
$$

relative to the measure $\mu\left[\chi^{\prime}\right]$, which we assume to be uniform, and subject to appropriate constraints. (Since we deal with infinitesimally short steps, the prior $Q$ turns out to be quite independent of the background measure $\mu$ ). The requirement that the field undergoes changes that are small and uncorrelated is implemented by imposing an infinite number of independent constraints, one per 
spatial point $x$,

$$
\left\langle\Delta \chi_{x}^{2}\right\rangle=\int D \chi^{\prime} Q\left[\chi^{\prime} \mid \chi\right]\left(\Delta \chi_{x}\right)^{2}=\kappa_{x},
$$

where $\Delta \chi_{x}=\chi_{x}^{\prime}-\chi_{x}$ and the $\kappa_{x}$ are small quantities. The result of maximizing (2) subject to (3) and normalization is a product of Gaussians

$$
Q\left[\chi^{\prime} \mid \chi\right] \propto \exp -\frac{1}{2} \int d x g_{x}^{1 / 2} \alpha_{x}\left(\Delta \chi_{x}\right)^{2},
$$

where $\alpha_{x}$ are the Lagrange multipliers associated to each constraint (3); the scalar density $g_{x}^{1 / 2}=\left(\operatorname{det} g_{i j}\right)^{1 / 2}$ is introduced so that $\alpha_{x}$ is a scalar field. For notational simplicity we write $d x^{\prime}$ instead of $d^{3} x^{\prime}$. To enforce the continuity of the motion we will eventually take the limit $\kappa_{x} \rightarrow 0$ which amounts to taking $\alpha_{x} \rightarrow \infty$.

\subsubsection{The Global Constraint}

The motion induced by the prior (4) leads to a rather simple diffusion process in the probabilities, in which the field variables evolve independently of each other. To model dynamics that exhibit correlations and are capable of demonstrating the full suite of quantum effects, such as the superposition of states, interference, and entanglement, however, we require additional structure. This is accomplished by imposing a single additional constraint that is non-local in space but local in configuration space, which involves the introduction of a drift potential $\phi[\chi]$ which is a scalar-valued functional defined over the configuration space $\mathcal{C}$. More explicitly, we impose

$$
\langle\Delta \phi\rangle=\int D \chi^{\prime} P\left[\chi^{\prime} \mid \chi\right] \int d x \Delta \chi_{x} \frac{\delta \phi[\chi]}{\delta \chi_{x}}=\kappa^{\prime},
$$

where we require $\kappa^{\prime} \rightarrow 0$. (Note that since $\chi_{x}$ and $\Delta \chi_{x}$ are scalars, in order that (5) be invariant under coordinate transformations of the surface, the derivative $\delta / \delta \chi_{x}$ must transform as a scalar density).

Before moving on, we discuss the central role of the drift potential in ED. As a set of dynamics of probabilities, ED brings together three ingredients. First, from an inference perspective, the dynamics consist of the updating of probabilities which must obey the rules of entropic inference. This requires information codified into constraints and the drift potential is the function that codifies such information. Second, in order to formulate a dynamical theory in which probabilities are generalized coordinates, it is only natural to attempt to identify the canonically conjugate generalized momenta and the corresponding symplectic structure. And third, from a purely geometric perspective, the trajectories we seek are curves on the space of probability distributions. For this statistical manifold it is natural to associate its tangent bundle. The fibers of such a bundle are spaces of velocity vectors tangent to all curves. It is also natural to consider the associated cotangent bundles of fibers as being the spaces of tangent covectors. As we shall see, ED brings together these three separate ingredients by identifying the constraint embodied in the drift potential functional with the momenta conjugate to the probabilities, with the cotangent vectors. More explicitly, we identify the three separate pairs: probability/constraint, coordinate/momentum, and point/covector. And as we shall see, eventually these three pairs are also identified with yet a fourth pair: the magnitude/phase of the wave function.

\subsection{The Transition Probability}

Next we maximize (1) subject to (5) and normalization. The multiplier $\alpha^{\prime}$ associated to the global constraint (5) turns out to have no influence on the dynamics: it can be absorbed into the yet undetermined drift potential $\alpha^{\prime} \phi \rightarrow \phi$, effectively setting $\alpha^{\prime}=1$. 
The result is a Gaussian transition probability distribution,

$$
P\left[\chi^{\prime} \mid \chi\right] \propto \exp -\frac{1}{2} \int d x g_{x}^{1 / 2} \alpha_{x}\left(\Delta \chi_{x}-\frac{1}{g_{x}^{1 / 2} \alpha_{x}} \frac{\delta \phi[\chi]}{\delta \chi_{x}}\right)^{2} .
$$

In previous work [26,27], $\alpha_{x}$ was chosen to be a spatial constant $\alpha$ to reflect the translational symmetry of flat space. Such a requirement, however, turns out to be inappropriate in the context of curved space-time. Instead, we follow $[28,29]$ in allowing $\alpha_{x}$ to remain a non-uniform spatial scalar. This will be a key element in developing our scheme for a local entropic time.

The form of (6) allows us to present a generic change,

$$
\Delta \chi_{x}=\left\langle\Delta \chi_{x}\right\rangle+\Delta w_{x}
$$

as resulting from an expected drift $\left\langle\Delta \chi_{x}\right\rangle$ plus Gaussian fluctuations $\Delta w_{x}$. Computing the expected short step for $\chi_{x}$ gives

$$
\left\langle\Delta \chi_{x}\right\rangle=\frac{1}{g_{x}^{1 / 2} \alpha_{x}} \frac{\delta \phi[\chi]}{\delta \chi_{x}}
$$

while the fluctuations $\Delta w_{x}$ satisfy,

$$
\left\langle\Delta w_{x}\right\rangle=0, \quad \text { and } \quad\left\langle\Delta w_{x} \Delta w_{x^{\prime}}\right\rangle=\frac{1}{g_{x}^{1 / 2} \alpha_{x}} \delta_{x x^{\prime}}
$$

Thus we see that while the expected step size is of order $\Delta \bar{\chi}_{x} \sim 1 / \alpha_{x}$, the fluctuations go as $\Delta w_{x} \sim 1 / \alpha_{x}^{1 / 2}$. Thus, for short steps, i.e., $\alpha_{x} \rightarrow \infty$, the fluctuations overwhelm the drift, resulting in a trajectory that is continuous but not, in general, differentiable. Such a model describes a Brownian motion in the field variables $\chi_{x}$.

\section{Some Notation}

The ED developed here deals with the coupled evolution of a quantum scalar field together with a classical dynamical background. The theories that allow such evolving geometries are often called instances of geometrodynamics. In typical geometrodynamics (see, e.g., [57-59]), the primary object of interest is the evolving three-metric $g_{i j}(x)$, whose dynamics must be suitably constrained so that the time evolution sweeps a four-dimensional space-time with metric ${ }^{4} g_{\mu v}(\mu, v, \ldots=0,1,2,3)$. Thus, despite the intrinsic dynamics at play, it is nonetheless appropriate to make reference to the enveloping space-time, if only as formal scaffolding that can be later removed.

It is therefore possible to assign coordinates $X^{\mu}$ to the space-time manifold. Furthermore, we deal with a space-time with globally hyperbolic topology, admitting a foliation by space-like surfaces $\{\sigma\}$. The embedding of such surfaces in space-time is defined by four scalar embedding functions $X^{\mu}\left(x^{i}\right)=X_{x}^{\mu}$. An infinitesimal deformation of the surface $\sigma$ to a neighboring surface $\sigma^{\prime}$ is determined by the deformation vector,

$$
\delta \xi_{x}^{\mu}=\delta \xi_{x}^{\perp} n_{x}^{\mu}+\delta \xi_{x}^{i} X_{i x}^{\mu}
$$

Here we have introduced $n^{\mu}$, which is the unit normal to the surface that is determined by the conditions $n_{\mu} n^{\mu}=-1$ and $\left.n_{\mu} X_{i x}^{\mu}=0\right)$, and where we have introduced $X_{i x}^{\mu}=\partial_{i x} X_{x}^{\mu}$, which are the space-time components of three-vectors tangent to $\sigma$. The normal and tangential components of $\delta \xi^{\mu}$, also known as the infinitesimal lapse and shift, are collectively denoted $\delta \xi_{x}^{A}=\left(\delta \xi_{x}^{\perp}, \delta \xi_{x}^{i}\right)$ and are given by

$$
\delta \xi_{x}^{\perp}=-n_{\mu x} \delta \xi_{x}^{\mu} \quad \text { and } \delta \xi_{x}^{i}=X_{\mu x}^{i} \delta \xi_{x}^{\mu}
$$


where $X_{\mu x}^{i}={ }^{4} g_{\mu v} g^{i j} X_{j x}^{v}$. Additionally, a particular deformation is defined by its components $\xi_{x}^{A}$, which allows us to speak unambiguously about applying the same deformations to different surfaces. Consequently, the very same deformation $\xi_{x}^{A}$ applied to different surfaces with distinct normal vectors $n_{x}^{\mu}$ will generally yield different deformation vectors $\xi_{x}^{\mu}$, as per Equation (9).

\section{Entropic Time}

In ED, entropic time is introduced as a tool for keeping track of the accumulation of many short steps. (For additional details on entropic time, see, e.g., [18]). Here we introduce a manifestly covariant notion of entropic time, along the lines of that in $[28,29]$.

\subsection{An Instant}

Central to the formulation of entropic time is the notion of an instant, which includes two main components. One is kinematic, the other informational. The former amounts to specifying a particular space-like surface. The latter consists of specifying the contents of the instant, namely, the information - the relevant probability distributions, drift potentials, geometries, etc.-which are necessary to generate the next instant.

\subsection{Ordered Instants}

Establishing the notion of an instant proves crucial because it supplies the structure necessary to inquire about the field $\chi_{x}$ at a moment of time. Equivalently, such a notion allows us to assign a probability distribution $\rho_{\sigma}[\chi]$ corresponding to the informational state of the field $\chi_{x}$ at an instant labeled by the surface $\sigma$.

Dynamics in ED, on the other hand, are constructed step-by-step, as a sequence of instants. Thus it is appropriate to turn our attention to the issue of updating from some distribution $\rho_{\sigma}[\chi]$ at some initial instant, to another distribution $\rho_{\sigma^{\prime}}[\chi]$ at a subsequent instant. Such dynamical information is encoded in the short-step transition probability from Equation (6), or better yet, the joint probability $P\left[\chi^{\prime}, \chi\right]=P\left[\chi^{\prime} \mid \chi\right] \rho_{\sigma}[\chi]$.

Application of the "sum rule" of probability theory to the joint distribution yields

$$
\rho_{\sigma^{\prime}}\left[\chi^{\prime}\right]=\int D \chi P\left[\chi^{\prime} \mid \chi\right] \rho_{\sigma}[\chi]
$$

The structure of Equation (11) is highly suggestive: if we interpret $\rho_{\sigma}[\chi]$ as being an initial state, then we can interpret $\rho_{\sigma^{\prime}}\left[\chi^{\prime}\right]$ as being posterior to it in the sense that it has taken into account the new information captured by the transition probability $P\left[\chi^{\prime} \mid \chi\right]$. Here we take this hint seriously and adopt Equation (11) as the equation that we seek for updating probabilities.

\subsection{Duration}

A final aspect of time to be addressed is the duration between instants. In doing so, one must distinguish between two separate issues. On one hand there is a natural notion of time that can be inherited from space-time itself, this being the local proper time $\delta \xi_{x}^{\perp}$ experienced by an observer at the point $x$ (see, e.g., [60]). On the other hand, however, notions of time and duration cannot themselves be divorced from those of dynamics and change. Indeed, the two are closely bound together.

Our strategy can be summarized by Wheeler's maxim [61]: “Time is defined so that motion looks simple." In Newtonian mechanics, for example, time is defined so as to simplify the motion of free particles; the prototype of a clock is a free particle that moves equal distances in equal times. In ED for short steps, the dynamics are dominated by fluctuations, Equation (9). Accordingly, the prototype of a 
clock is provided by the fluctuations of the field. Since the specification of the time interval is achieved by an appropriate choice of multipliers alpha, we proceed by setting

$$
\alpha_{x}=\frac{1}{\delta \xi_{x}^{\perp}} \quad \text { so that }\left\langle\Delta w_{x} \Delta w_{x^{\prime}}\right\rangle=\frac{\delta \xi_{x}^{\perp}}{g_{x}^{1 / 2}} \delta_{x x^{\prime}}
$$

With this the transition probability, Equation (6) resembles a Wiener process, albeit in a rather unfamiliar context involving the propagation of fields on curved space. Equation (12) shows that at each point $x$ time is defined by a local clock so that fluctuations as measured by the variance increase by equal amounts in equal time delta $\xi_{x}^{\perp}$.

\subsection{The Local-Time Diffusion Equations}

The dynamics expressed in integral form by (11) and (12) can be rewritten in differential form. The result is $[28,29]$,

$$
\delta \rho_{\sigma}[\chi]=\int d x \frac{\delta \rho_{\sigma}[\chi]}{\delta \xi_{x}^{\perp}} \delta \xi_{x}^{\perp}=-\int d x \frac{1}{g_{x}^{1 / 2}} \frac{\delta}{\delta \chi_{x}}\left(\rho_{\sigma}[\chi] \frac{\delta \Phi_{\sigma}[\chi]}{\delta \chi_{x}}\right) \delta \xi_{x}^{\perp}
$$

where we have introduced

$$
\Phi_{\sigma}[\chi]=\phi_{\sigma}[\chi]-\log \rho_{\sigma}^{1 / 2}[\chi]
$$

which we refer to as the phase functional.

For arbitrary choices of the infinitesimal lapse $\delta \xi_{x}^{\perp}$ we obtain an infinite set of local equations, one for each spatial point

$$
\frac{\delta \rho_{\sigma}}{\delta \xi_{x}^{\perp}}=-\frac{1}{g_{x}^{1 / 2}} \frac{\delta}{\delta \chi_{x}}\left(\rho_{\sigma} \frac{\delta \Phi_{\sigma}}{\delta \chi_{x}}\right)
$$

To interpret these local equations, consider again the variation given in Equation (13). In the special case where both surfaces $\sigma$ and $\sigma^{\prime}$ happen to be flat, then $g_{x}^{1 / 2}=1$ and $\delta \xi_{x}^{\perp}=d t$ are both spatial constants and Equation (13) becomes equivalent to

$$
\frac{\partial \rho_{t}[\chi]}{\partial t}=-\int d x \frac{\delta}{\delta \chi_{x}}\left(\rho_{t}[\chi] \frac{\delta \Phi_{t}[\chi]}{\delta \chi_{x}}\right) .
$$

We recognize this $[26,27]$ as a diffusion or Fokker-Planck equation written as a continuity equation for the flow of probability in configuration space $\mathcal{C}$. This suggests identifying the

$$
V_{x}=\frac{1}{g_{x}^{1 / 2}} \frac{\delta \Phi_{\sigma}[\chi]}{\delta \chi_{x}}
$$

that appears in Equation (15) as the velocity of the probability current which is valid for curved and flat spaces alike. Accordingly, we will refer to (15) as the "local-time Fokker-Planck" equations (LTFP).

\subsection{The Phase Functional}

Note that the phase functional $\Phi_{\sigma}$ is defined entirely through (14) and thus requires no additional interpretation. Nevertheless, one can be provided. A key aspect of ED is that we model the dynamics of probabilities, rather than the corresponding microstates. Therefore, while we do introduce a symplectic structure and fully Hamiltonian formalism in ED, it is for the probability $\rho_{\sigma}$ and its eventual conjugate variable $\Phi_{\sigma}$, not for the ontic field $\chi_{x}$, which, strictly speaking, has no equivalent notion of a conjugate momentum. Still, an analogous concept can be introduced in ED using the phase functional $\Phi_{\sigma}$ through $P_{x}=\delta \Phi_{\sigma} / \delta \chi_{x}$. As shown in [29], the expected values of $\chi_{x}$ and $P_{x}$ do, in fact, satisfy a Poisson bracket 
that is very reminiscent of the canonical Poisson bracket relations of classical physics. This suggests identifying $P_{x}$ itself as a type of momentum, which very much resembles the notion of momentum familiar from the Hamilton-Jacobi formulation of classical physics.

\section{The Structure of Surface Deformations}

Starting with Dirac [42,57] and developed more fully by Hojman, Kuchař, and Teitelboim, a chief contribution of the DHKT program was the recognition that covariant dynamical theories had a rich structure that could be traced to the kinematics of surface deformations. Such structure can, however, itself be studied independently of any particular dynamics being considered. We give a brief review of the subject following the presentations of Kuchař [45] and Teitelboim [46].

For simplicity, we consider a generic functional $T[X(x)]$ that assigns a real number to every surface defined by the four embedding variables $X^{\mu}(x)$. The variation in the functional $\delta T$ resulting from an arbitrary deformation $\delta \xi_{x}^{A}$ has the form

$$
\delta T=\int d x \delta \xi_{x}^{\mu} \frac{\delta T}{\delta \xi_{x}^{\mu}}=\int d x\left(\delta \xi_{x}^{\perp} G_{\perp x}+\delta \xi_{x}^{i} G_{i x}\right) T
$$

where

$$
G_{\perp x}=\frac{\delta}{\delta \xi_{x}^{\perp}}=n_{x}^{\mu} \frac{\delta}{\delta X_{x}^{\mu}} \quad \text { and } \quad G_{i x}=\frac{\delta}{\delta \xi_{x}^{i}}=X_{i x}^{\mu} \frac{\delta}{\delta X_{x}^{\mu}}
$$

are the generators of normal and tangential deformations respectively. The generators of deformations $\delta / \delta \xi_{x}^{A}$ form a non-holonomic basis. Thus, unlike the vectors $\delta / \delta X_{x}^{\mu}$, which form a coordinate basis and therefore commute, the generators of deformations have a non-vanishing commutator "algebra" given by

$$
\frac{\delta}{\delta \xi_{x}^{A}} \frac{\delta}{\delta \xi_{x^{\prime}}^{B}}-\frac{\delta}{\delta \xi_{x^{\prime}}^{B}} \frac{\delta}{\delta \xi_{x}^{A}}=\int d x^{\prime \prime} \kappa^{C}{ }_{B A}\left(x^{\prime \prime} ; x^{\prime}, x\right) \frac{\delta}{\delta \xi_{x^{\prime \prime}}^{C}}
$$

where $\kappa^{C}{ }_{B A}$ are the "structure constants" of the "group" of deformations.

The previous quotes in "group" and "algebra" are a reminder that strictly, the set of deformations do not form a group. The composition of two successive deformations is itself a deformation, of course, but it also depends on the surface to which the first deformation is applied. As we will see below, the "structure constants" $\kappa^{C}{ }_{B A}$ are not constant; they depend on the metric $g_{i j}$ of the initial surface. (In a dynamical approach to gravity the metric is itself a functional of the canonical variables. Thus its appearance in the "algebra" of deformations is in part responsible for the rich structure of geometrodynamics).

The calculation of $\kappa_{B A}^{C}$ is given in [45,46]. The key idea is that of embeddability, which proceeds as follows. Consider performing two successive infinitesimal deformations $\delta \xi^{A}$ followed by $\delta \eta^{A}$ on an initial surface $\sigma: \sigma \stackrel{\delta \xi}{\rightarrow} \sigma_{1} \stackrel{\delta \eta}{\rightarrow} \sigma^{\prime}$. Performing now the very same deformations in the opposite order $\sigma \stackrel{\delta \eta}{\rightarrow} \sigma_{2} \stackrel{\delta \xi}{\rightarrow} \sigma^{\prime \prime}$ yields a final surface $\sigma^{\prime \prime}$ that, in general, differs from $\sigma^{\prime}$. The key point is that since both $\sigma^{\prime}$ and $\sigma^{\prime \prime}$ are embedded in the very same space-time, then there exists a third deformation $\delta \zeta^{A}$ that relates the two: $\sigma^{\prime} \stackrel{\delta \zeta}{\rightarrow} \sigma^{\prime \prime}$.

As shown by Teitelboim [46], however, the compensating deformation is not at all arbitrary, but can be determined entirely by geometrical arguments:

$$
\delta \zeta_{x^{\prime \prime}}^{C}=\int d x \int d x^{\prime} \kappa_{A B}^{C}\left(x^{\prime \prime} ; x, x^{\prime}\right) \delta \xi_{x}^{A} \delta \eta_{x^{\prime}}^{B}
$$


where the only non-vanishing $\kappa s$ have the form

$$
\begin{aligned}
\kappa_{i \perp}^{\perp}\left(x^{\prime \prime} ; x, x^{\prime}\right) & =-\kappa_{\perp i}^{\perp}\left(x^{\prime \prime} ; x^{\prime}, x\right)=-\delta\left(x^{\prime \prime}, x\right) \partial_{i x^{\prime \prime}} \delta\left(x^{\prime \prime}, x^{\prime}\right) \\
\kappa_{i j}^{k}\left(x^{\prime \prime} ; x, x^{\prime}\right) & =-\kappa_{j i}^{k}\left(x^{\prime \prime} ; x^{\prime}, x\right) \\
& =\delta\left(x^{\prime \prime}, x\right) \partial_{i x^{\prime \prime}} \delta\left(x^{\prime \prime}, x^{\prime}\right) \delta_{j}^{k}-\delta\left(x^{\prime \prime}, x\right) \partial_{j x^{\prime \prime}} \delta\left(x^{\prime \prime}, x^{\prime}\right) \delta_{i}^{k} \\
\kappa_{\perp \perp}^{i}\left(x^{\prime \prime} ; x, x^{\prime}\right) & =-\kappa_{\perp \perp}^{i}\left(x^{\prime \prime} ; x^{\prime}, x\right) \\
& =-g^{i j}\left(x^{\prime \prime}\right)\left(\delta\left(x^{\prime \prime}, x^{\prime}\right) \partial_{j x^{\prime \prime}} \delta\left(x^{\prime \prime}, x\right)-\delta\left(x^{\prime \prime}, x\right) \partial_{j x^{\prime \prime}} \delta\left(x^{\prime \prime}, x^{\prime}\right)\right)
\end{aligned}
$$

Identification of the $\kappa s$ implies that the commutator in Equation (19) satisfies the "algebra" [45,46],

$$
\left[G_{A x}, G_{B x^{\prime}}\right]=\int d x^{\prime \prime} \kappa_{A B}^{C}\left(x^{\prime \prime} ; x, x^{\prime}\right) G_{C x^{\prime \prime}},
$$

which can now be written more explicitly as

$$
\begin{aligned}
{\left[G_{\perp x}, G_{\perp x^{\prime}}\right] } & =-\left(g_{x}^{i j} G_{j x}+g_{x^{\prime}}^{i j} G_{j x^{\prime}}\right) \partial_{i x} \delta\left(x, x^{\prime}\right), \\
{\left[G_{i x}, G_{\perp x^{\prime}}\right] } & =-G_{\perp x} \partial_{i x} \delta\left(x, x^{\prime}\right) \\
{\left[G_{i x}, G_{j x^{\prime}}\right] } & =-G_{i x^{\prime}} \partial_{j x} \delta\left(x, x^{\prime}\right)-G_{j x} \partial_{i x} \delta\left(x, x^{\prime}\right),
\end{aligned}
$$

with all other brackets vanishing.

\section{Entropic Geometrodynamics}

In entropic dynamics, evolution is driven by information codified into constraints. Entropic geometrodynamics, it follows, consist of dynamics driven by a specific choice of constraints, which we discuss here. In $[28,29]$, quantum field theory in a curved space-time (QFTCS) was derived under the assumption that the geometry remains fixed. But such assumptions, we know, should break down when one considers states describing a non-negligible concentration of energy and momentum. Thus we must revise our constraints appropriately. A natural way to proceed is thus to allow the geometry itself to take part in the dynamical process: the geometry affects $\rho_{\sigma}[\chi]$ and $\phi_{\sigma}[\chi]$; they then act back on the geometry, and so forth. Our goal here is to make this interplay concrete.

\subsection{The Canonical Updating Scheme}

A natural question that arises from the above discussion is how to implement the update of the geometry and drift potential $\phi_{\sigma}[\chi]$. Such a task involves two steps. The first is the proper identification of variables for describing the evolving geometry, while the other is the specific manner in which this joint system of variables, including the drift potential $\phi_{\sigma}[\chi]$, is updated. Fortunately, the two challenges can be dealt with quite independently of each other.

In devising a covariant scheme for updating, we draw primarily from the work of IAC [28,29]. To review briefly, a primary assumption in the IAC approach was the adoption of a canonical framework for governing the coupled dynamics of $\rho_{\sigma}$ and $\phi_{\sigma}$, expressed more conveniently through the transformed variable $\Phi_{\sigma}$. Although this is certainly a strong assumption, it is one that has some justification. On one front, it can be argued that canonical structures seem to have a rather natural place in ED; arising from conservation laws in [22,27] and alternatively from symmetry considerations [23]. However, from another perspective entirely, the use of a canonical formalism in ED can also be traced to more pragmatic concerns, as it allows one to borrow from a roster of covariant canonical techniques designed in the context of classical physics (see, e.g., [43]), but deployed for the purposes of ED. These together suggest that we view the canonical setting, and the symplectic symmetries that undergird it, as a central criterion for updating in ED. 


\subsection{The Canonical Variables}

Crucial to our updating scheme is an appropriate choice of variables. We pursue a conservative approach in which $\rho_{\sigma}$ and $\Phi_{\sigma}$ are packaged together as canonically conjugate variables following the prescription detailed in IAC [29]. The nontrivial task of choosing the geometric variables has long been the subject of a lively debate. To review briefly, there are, of course, the original attempts at geometrodynamics from Dirac [57] as well as Arnowitt, Deser, and Misner (ADM) [58] that start from the Einstein-Hilbert action and take the metric $g_{i j}$ as the fundamental building block. Somewhat more recently, due in part to the modern success of gauge theories, there has been some interest in taking, not the metric $g_{i j}$, but the Levi-Civita connection $\Gamma_{j k}^{i}$, as the fundamental gravitational object (see, e.g., [62]). In a similar spirit is the well-known discovery of Ashtekar [63], which uses triads and spin connections to rewrite GR.

Among these various approaches, however, the pioneering efforts of Hojman, Kuchař, and Teitelboim (HKT) [44] prove to be of special interest, as their work centers a purely canonical approach on the dynamics of geometry. Following HKT, it is possible to take the six components of the metric $g_{i j}$ to be the starting point for a geometrodynamics. The argument for this is grounded in simplicity. If one assumes, as they do, that evolution in local time should mirror the structure of space-time deformations, then the metric appearing on the right hand side of (23) for the "structure constant" $\kappa_{\perp \perp}^{i}$ must necessarily be a functional of the canonical variables. Clearly, this is most easily satisfied if the metric is itself a canonical variable.

To complete the canonical framework, however, we must also introduce six variables $\pi^{i j}$ that are canonically conjugates to the $g_{i j}$. These variables, the conjugate momenta, are themselves defined through the canonical Poisson bracket relations,

$$
\begin{aligned}
\left\{g_{i j x}, g_{k l x^{\prime}}\right\} & =\left\{\pi_{x}^{i j}, \pi_{x^{\prime}}^{k l}\right\}=0 \\
\left\{g_{i j x}, \pi_{x^{\prime}}^{k l}\right\} & =\delta_{i j}^{k l} \delta\left(x, x^{\prime}\right)=\frac{1}{2}\left(\delta_{i}^{k} \delta_{j}^{l}+\delta_{j}^{k} \delta_{i}^{l}\right) \delta\left(x, x^{\prime}\right) .
\end{aligned}
$$

(In particular, we do not assume the existence of a Legendre transformation from $\pi^{i j}$ to the "velocity" of the metric $g_{i j}$ ).

That such relations are satisfied is made manifest by writing the Poisson brackets in local coordinates

$$
\begin{aligned}
\{F, G\} & =\int d x\left(\frac{\delta F}{\delta g_{i j x}} \frac{\delta G}{\delta \pi_{x}^{i j}}-\frac{\delta G}{\delta g_{i j x}} \frac{\delta F}{\delta \pi_{x}^{i j}}\right) \\
& +\int D \chi\left(\frac{\tilde{\delta} F}{\tilde{\delta} \rho[\chi]} \frac{\tilde{\delta} G}{\tilde{\delta} \Phi[\chi]}-\frac{\tilde{\delta} G}{\tilde{\delta} \rho[\chi]} \frac{\tilde{\delta} F}{\tilde{\delta} \Phi[\chi]}\right),
\end{aligned}
$$

where $F$ and $G$ are some arbitrary functionals of the phase space variables. (Note that $\pi_{x}^{i j}$ must be a tensor density for the Poisson bracket to transform appropriately under a change of variables of the surface).

\section{The Canonical Structure of Space-Time}

Fully covariant dynamics require that the updating in local time of all dynamical variables be consistent with the kinematics of surface deformations. Thus, the requirement that the deformed surfaces remain embedded in space-time, which amounts to imposing foliation invariance, translates into a consistency requirement of path independence: if the evolution from an initial instant into a final instant can occur along different paths, then all these paths must lead to the same final values for all dynamical quantities. The approach we adopt for quantum fields coupled to dynamical classical gravity builds on previous work by HKT [44] for classical geometrodynamics, and by IAC [28,29] for quantum field theory in a non-dynamical space-time. 
Within this scheme the evolution of an arbitrary functional $F$ of the canonical variables is generated by application of a set of local Hamiltonians according to

$$
\delta F=\int d x\left\{F, H_{A x}\right\} \delta \xi_{x}^{A}=\int d x\left(\left\{F, H_{\perp x}\right\} \delta \xi_{x}^{\perp}+\left\{F, H_{i x}\right\} \delta \xi_{x}^{i}\right)
$$

where parameters $\delta \xi_{x}^{A}$ with $A=(\perp, i=1,2,3)$ describe an infinitesimal deformation, as per Equations (9) and (10), and $H_{A x}$ are the corresponding generators. (Defined in this way, the $H_{A x}$ turn out to be tensor densities).

\subsection{Path Independence}

The implementation of path independence $[45,46]$ then rests on the idea that the Poisson brackets of the generators $H_{A x}$ form an "algebra" that closes in the same way, that is, with the same "structure constants," as the "algebra" of deformations in Equations (25)-(27).

$$
\begin{aligned}
\left\{H_{\perp x}, H_{\perp x^{\prime}}\right\} & =\left(g_{x}^{i j} H_{j x}+g_{x^{\prime}}^{i j} H_{j x^{\prime}}\right) \partial_{i x} \delta\left(x, x^{\prime}\right), \\
\left\{H_{i x}, H_{\perp x^{\prime}}\right\} & =H_{\perp x} \partial_{i x} \delta\left(x, x^{\prime}\right), \\
\left\{H_{i x}, H_{j x^{\prime}}\right\} & =H_{i x^{\prime}} \partial_{j x} \delta\left(x, x^{\prime}\right)+H_{j x} \partial_{i x} \delta\left(x, x^{\prime}\right) .
\end{aligned}
$$

We conclude this section with two remarks. First, we note that these equations have not been derived. Indeed, imposing (25)-(27) as strong constraints constitutes the definition of what we mean by imposing consistency between the updating of dynamical variables and the kinematics of surface deformations. But this is not enough. As discussed in detail by Teitelboim [46] and HKT [44], one achieves path independence by requiring that the initial values of the canonical variables be restricted to satisfy the weak constraints

$$
H_{\perp x} \approx 0 \text { and } H_{i x} \approx 0
$$

While it is beyond the scope of this paper to explore the full consequences of these weak constraints, we merely point out that formally their origin is traced to the fact that $\kappa_{\perp \perp}^{i}$ in Equation (23) depends on the metric and therefore it is not a true structure "constant." More physically, the constraints represent the fact that the canonical variables $g_{i j}$ and $\pi^{i j}$ are redundant because they represent the true dynamical degrees of freedom plus additional kinematical variables that allow us the freedom to choose space-time coordinates; and, as discussed by Kuchař [45], separating these dynamical and kinematical variables is not an easy task. Furthermore, once satisfied on an initial surface $\sigma$ the dynamics will be such as to preserve these constraints for all subsequent surfaces of the foliation.

We also note that it is only by virtue of relating the conditions of "integrability" to those of "embeddability" that we can interpret the role of the local Hamiltonians $H_{\perp x}$ and $H_{i x}$ in relation to space and time. This is a crucial and highly non-trivial step. It is only once this is established that we can interpret $H_{\perp x}$ as a scalar density that is responsible for genuine dynamical evolution and $H_{i x}$ as a vector density that generates spatial diffeomorphisms; the former is called the super-Hamiltonian, while the latter is the so-called super-momentum.

\section{The Canonical Representation}

We now turn our attention to the local Hamiltonian generators $H_{A x}$, and more specifically, we look to provide explicit expressions for these generators in terms of the canonical variables. This problem was solved in the context of purely classical geometrodynamics (with or without sources) by HKT in [44]. Here we aim to apply their techniques and methodology to a different problem: geometrodynamics driven by "quantum" sources. Fortunately, a considerable portion of the HKT formalism can be directly adopted for our purposes. 


\subsection{The Super-Momentum}

To determine the generators $H_{A x}\left[\rho, \Phi ; g_{i j}, \pi^{i j}\right]$ it is easiest to begin with the so-called super-momentum $H_{i x}\left[\rho, \Phi ; g_{i j}, \pi^{i j}\right]$. This is largely because the function of this generator is well understood: it pushes the canonical variables along the surface they reside on. Since there is no motion "normal" to the surface, the action of this generator is purely kinematical.

Following HKT [44], consider an infinitesimal tangential deformation such that a point originally labeled by $x^{i}$ is carried to the point previously labeled by $x^{i}+\delta \xi^{i}$. This will induce a corresponding change in any dynamical variables $F$ defined on that surface, $F \rightarrow F+\delta F$. This change $\delta F$ can then be computed in two distinct ways, which, of course, must agree. One is by calculating the Lie derivative along $\delta \xi$

$$
\delta F=£_{\delta \xi} F
$$

and the other is using the super-momentum $H_{i x}$, so that

$$
\delta F=\int d x\left\{F, H_{i x}\right\} \delta \xi_{x}^{i} .
$$

\subsubsection{Gravitational Super-Momentum}

A straightforward example of this is shown for the metric $g_{i j}(x)=g_{i j x}$, which is a rank $(0,2)$ tensor. Its Lie derivative is given by [64]

$$
£_{\delta \xi} g_{i j}=\partial_{k} g_{i j} \delta \xi_{x}^{k}+g_{i k} \partial_{j} \delta \xi_{x}^{k}+g_{k j} \partial_{i} \delta \xi_{x}^{k}
$$

Alternatively, by using the Poisson brackets we obtain

$$
\delta g_{i j x}=\int d x^{\prime}\left\{g_{i j x}, H_{k x^{\prime}}\right\} \delta \xi_{x^{\prime}}^{k}=\int d x^{\prime} \frac{\delta H_{k x^{\prime}}}{\delta \pi_{x}^{i j}} \delta \xi_{x^{\prime}}^{k}
$$

Comparing the two and using the fact that $\delta \xi_{x}^{i}$ is arbitrary yields

$$
\frac{\delta H_{k x^{\prime}}}{\delta \pi_{x}^{i j}}=\partial_{k x} g_{i j x} \delta\left(x, x^{\prime}\right)+g_{i k x} \partial_{j x} \delta\left(x, x^{\prime}\right)+g_{k j x} \partial_{i x} \delta\left(x, x^{\prime}\right)
$$

The delta functions imply that $H_{i x}$ is local in the momentum $\pi^{i j}$.

To fix the dependence on $\pi^{i j}$, in fact, we can use a similar argument as above. Recalling that $\pi^{i j}(x)=\pi_{x}^{i j}$ is a rank $(2,0)$ tensor density of weight one, we find that

$$
£_{\delta \xi} \pi^{i j}=\partial_{k x}\left(\pi^{i j} \delta \xi_{x}^{k}\right)-\pi^{i k} \partial_{k x} \delta \xi_{x}^{j}-\pi^{k j} \partial_{k x} \delta \xi_{x}^{i} .
$$

The same equation can be obtained through the use of a Hamiltonian,

$$
\delta \pi_{x}^{i j}=\int d x^{\prime}\left\{\pi_{x}^{i j}, H_{k x^{\prime}}\right\} \delta \xi_{x^{\prime}}^{k}=-\int d x^{\prime} \frac{\delta H_{k x^{\prime}}}{\delta g_{i j x}} \delta \xi_{x^{\prime}}^{k}
$$

so long as $H_{i x}$ satisfies

$$
-\frac{\delta H_{k x^{\prime}}}{\delta g_{i j x}}=\partial_{k x}\left(\pi_{x}^{i j} \delta\left(x, x^{\prime}\right)\right)-\pi_{x}^{i l} \partial_{l x} \delta\left(x, x^{\prime}\right) \delta_{k}^{j}-\pi_{x}^{l j} \partial_{l x} \delta\left(x, x^{\prime}\right) \delta_{k}^{i} .
$$

Integrating these equations for $H_{i x}$ yields

$$
H_{i x}=H_{i x}^{G}+\tilde{H}_{i x}
$$


where

$$
H_{i x}^{G}=-2 \partial_{j x}\left(\pi^{j k} g_{i k}\right)+\pi^{j k} \partial_{i x} g_{j k}
$$

is called the gravitational super-momentum, and the functional $\tilde{H}_{i x}=\tilde{H}_{i x}[\rho, \Phi]$ is, at the moment, just an integration "constant."

Some of these expressions can be simplified by introducing the covariant derivative $\nabla_{i}$. For example, using $\nabla_{k} g_{i j}=0$ we have

$$
£_{\delta \xi} g_{i j x}=g_{j k} \nabla_{i} \delta \xi_{x}^{k}+g_{i k} \nabla_{j} \delta \xi_{x}^{k}
$$

and

$$
£_{\delta \xi} \pi_{x}^{i j}=\nabla_{k}\left(\pi^{i j} \delta \xi_{x}^{k}\right)-\pi^{i k} \nabla_{k} \delta \xi_{x}^{j}-\pi^{k j} \nabla_{k} \delta \xi_{x}^{i}
$$

The gravitational super-momentum $H_{i x}^{G}$ also takes the particularly simple form

$$
H_{i x}^{G}=-2 g_{i k} \nabla_{j} \pi^{j k}=-2 \nabla_{j}\left(\pi^{j k} g_{i k}\right)=-2 \nabla_{j} \pi_{i}^{j} .
$$

\subsubsection{The "Matter" Super-Momentum}

Next, we turn to the response of the variables $\rho_{\sigma}[\chi]$ and $\Phi_{\sigma}[\chi]$ under a relabeling of the surface coordinates $x^{i} \rightarrow x^{i}+\delta \xi_{x}^{i}$. As the coordinates are shifted, so too are the fields defined upon that surface so that

$$
\chi_{x} \rightarrow \chi_{x}+£_{\delta \xi} \chi_{x} \quad \text { where } £_{\delta \xi} \chi_{x}=\partial_{i x} \chi_{x} \delta \xi_{x}^{i}
$$

is the Lie derivative for a scalar $\chi_{x}$. This induces a change in the probability, given by

$$
\delta \rho_{\sigma}[\chi] \equiv \rho_{\sigma}\left[\chi+£_{\delta \xi} \chi_{x}\right]-\rho_{\sigma}[\chi]=\int d x \frac{\delta \rho_{\sigma}[\chi]}{\delta \chi_{x}} \partial_{i x} \chi_{x} \delta \xi_{x}^{i} .
$$

Alternatively, this same variation can be computed using the canonical framework

$$
\delta \rho_{\sigma}[\chi]=\int d x\left\{\rho_{\sigma}[\chi], H_{i x}\right\} \delta \xi_{x}^{i} .
$$

If we insert the super-momentum $H_{i x}$ given in Equation (40), we notice that only the $\tilde{H}_{i x}$ piece will contribute. And so we have

$$
\delta \rho_{\sigma}[\chi]=\int d x\left\{\rho_{\sigma}[\chi], \tilde{H}_{i x}\right\} \delta \xi_{x}^{i}=\int d x \frac{\tilde{\delta} \tilde{H}_{i x}}{\tilde{\delta} \Phi_{\sigma}[\chi]} \delta \xi_{x}^{i}
$$

which for arbitrary $\delta \xi_{x}^{i}$ requires that

$$
\frac{\tilde{\delta} \tilde{H}_{i x}}{\tilde{\delta} \Phi_{\sigma}[\chi]}=\frac{\delta \rho_{\sigma}[\chi]}{\delta \chi_{x}} \partial_{i x} \chi_{x}
$$

A similar argument for $\Phi_{\sigma}[\chi]$ shows that we must also have

$$
-\frac{\tilde{\delta} \tilde{H}_{i x}}{\tilde{\delta} \rho_{\sigma}[\chi]}=\frac{\delta \Phi_{\sigma}[\chi]}{\delta \chi_{x}} \partial_{i x} \chi_{x}
$$


so that

$$
\tilde{H}_{i x}=-\int D \chi \rho_{\sigma} \frac{\delta \Phi_{\sigma}}{\delta \chi_{x}} \partial_{i x} \chi_{x} .
$$

Thus the total super-momentum, Equation (40),

$$
H_{i x}=-2 \nabla_{j} \pi_{i}^{j}-\int D \chi \rho_{\sigma} \frac{\delta \Phi_{\sigma}}{\delta \chi_{x}} \partial_{i x} \chi_{x}
$$

contains two pieces, which we refer to as the gravitational and "matter" contributions, respectively. (The division of generators into gravitational and "matter" pieces established by DHKT is, strictly speaking, an abuse of language. The variables $\rho_{\sigma}$ and $\Phi_{\sigma}$ that constitute "matter" are more properly understood as describing the statistical state of the material field $\chi_{x}$. Nonetheless, we stick with the convention as a useful shorthand). Inspection of (51) confirms that there is no gravitational dependence in the "matter" side, or "matter" dependence on the gravitational side, that is,

$$
H_{i x}=H_{i x}^{G}\left[g_{i j}, \pi^{i j}\right]+\tilde{H}_{i x}[\rho, \Phi] .
$$

By Equation (35) it is, of course, also understood that $H_{i x}$ is subject to the constraint

$$
H_{i x} \approx 0
$$

Finally, although $H_{i x}$ was obtained, in essence, independently of the Poisson bracket (34) which relates two tangential deformations, it nonetheless satisfies it automatically. It is therefore appropriate to view $H_{i x}$ as being completely determined; this is important as it allows us to treat Equation (32) as a set of equations for $H_{\perp x}$ in terms of the known $H_{i x}$.

\subsection{The Super-Hamiltonian}

We now turn our attention to the generator $H_{\perp x}$ of local time evolution. Following Teitelboim [47], it is useful to decompose $H_{\perp x}$ into two distinct pieces

$$
H_{\perp x}=H_{\perp x}^{G}\left[g_{i j}, \pi^{i j}\right]+\tilde{H}_{\perp x}\left[\rho, \Phi ; g_{i j}, \pi^{i j}\right],
$$

consisting of a gravitational piece $H_{\perp x}^{G}$ depending only on the gravitational variables, and a "matter" contribution that we suggestively denote by $\tilde{H}_{\perp x}$, called the gravitational and "matter" super-Hamiltonians, respectively.

As noted by Teitelboim, we make no assumptions in writing $H_{\perp x}$ in this way since we can always, by construction, identify a piece of the super-Hamiltonian that updates the geometry alone. The "matter" super-Hamiltonian is then just defined as the difference between the total and gravitational pieces. Nonetheless, given this splitting, we do make the following simplifying assumption: we require the "matter" super-Hamiltonian $\tilde{H}_{\perp x}$ to be independent of the gravitational momentum $\pi^{i j}$, i.e.,

$$
\tilde{H}_{\perp x}=\tilde{H}_{\perp x}\left[\rho, \Phi ; g_{i j}\right]
$$

so that the super-Hamiltonian takes the form

$$
H_{\perp x}=H_{\perp x}^{G}\left[g_{i j}, \pi^{i j}\right]+\tilde{H}_{\perp x}\left[\rho, \Phi ; g_{i j}\right] .
$$

With this assumption in hand, it is possible to prove [47] that the metric appears in $\tilde{H}_{\perp x}$ only as a local function of $g_{i j}$. That is, no derivatives of the metric are allowed, nor are any other complicated 
functional dependencies; due to this fact, this was referred to as the non-derivative coupling assumption by Teitelboim.

\subsubsection{Modified Poisson Brackets}

A particularly appealing aspect of this separation into gravitational and "matter" pieces is that the Poisson bracket relations (32)-(34) also split along similar lines. In fact, insert the decomposed generators $H_{A x}$ from Equations (52) and (55) into the Poisson bracket relations (32)-(34). We find that the gravitational generators $H_{A x}^{G}=\left(H_{\perp x}^{G}, H_{i x}^{G}\right)$ must satisfy a set of Poisson brackets

$$
\begin{aligned}
\left\{H_{\perp x^{\prime}}^{G}, H_{\perp x^{\prime}}^{G}\right\} & =\left(g_{x}^{i j} H_{j x}^{G}+g_{x^{\prime}}^{i j} H_{j x^{\prime}}^{G}\right) \partial_{i x} \delta\left(x, x^{\prime}\right), \\
\left\{H_{i x}^{G}, H_{\perp x^{\prime}}^{G}\right\} & =H_{\perp x}^{G} \partial_{i x} \delta\left(x, x^{\prime}\right), \\
\left\{H_{i x}^{G}, H_{j x^{\prime}}^{G}\right\} & =H_{i x^{\prime}}^{G} \partial_{j x} \delta\left(x, x^{\prime}\right)+H_{j x}^{G} \partial_{i x} \delta\left(x, x^{\prime}\right),
\end{aligned}
$$

which have closing relations identical to those of (32)-(34).

The "matter" generators, which we collectively denote by $\tilde{H}_{A x}$, satisfy a somewhat modified set of brackets

$$
\begin{aligned}
\left\{\tilde{H}_{\perp x}, \tilde{H}_{\perp x^{\prime}}\right\} & =\left(g_{x}^{i j} \tilde{H}_{j x}+g_{x^{\prime}}^{i j} \tilde{H}_{j x^{\prime}}\right) \partial_{i x} \delta\left(x, x^{\prime}\right), \\
\left\{H_{i x}, \tilde{H}_{\perp x^{\prime}}\right\} & =\tilde{H}_{\perp x} \partial_{i x} \delta\left(x, x^{\prime}\right), \\
\left\{\tilde{H}_{i x}, \tilde{H}_{j x^{\prime}}\right\} & =\tilde{H}_{i x^{\prime}} \partial_{j x} \delta\left(x, x^{\prime}\right)+\tilde{H}_{j x} \partial_{i x} \delta\left(x, x^{\prime}\right) .
\end{aligned}
$$

Here we call attention to the fact that Equation (60) contains the total tangential generator $H_{i x}$, not just $\tilde{H}_{i x}$. This alteration occurs because the "matter" super-Hamiltonian depends on the metric. That is, if we want to shift $\tilde{H}_{\perp x}$ along a given surface, we must shift the variables $(\rho, \Phi)$, as well as the metric $g_{i j}$; hence $H_{i x}$, not just $\tilde{H}_{i x}$, must appear.

Modulo the small distinction arising in Equation (60), we see that the gravitational and "matter" sectors decouple such that each piece forms an independent representation of the "algebra" of surface deformations. From a strategic point of view, the separation means that we can solve the Poisson bracket relations for geometry and "matter" independently of one another.

\subsubsection{The "Matter" Super-Hamiltonian}

Our goal is to identify a family of ensemble super-Hamiltonians $\tilde{H}_{\perp x}\left[\rho, \Phi ; g_{i j}\right]$ that are consistent with the Poisson brackets (59)-(61). Let us briefly outline our approach. Thus far, we have completely determined the correct form of the "matter" super-momentum $\tilde{H}_{i x}$ which is consistent with (61). Moreover, the relation (60) merely implies that $\tilde{H}_{\perp x}$ transforms as a scalar density under a spatial diffeomorphism. Thus we are left only to satisfy the first Poisson bracket (59) for the unknown $\tilde{H}_{\perp x}$.

In addition to these considerations, however, the ED approach itself imposes additional constraints of a fundamental nature on the allowed $H_{\perp x}$, or more specifically $\tilde{H}_{\perp x}$. This is because, in ED, the introduction of a symplectic structure and its corresponding Hamiltonian formalism is not meant to replace the entropic updating methods that yield the LTFP equations, but to augment them, appropriately. As a consequence, we demand, as a matter of principle, that the "matter" super-Hamiltonian $\tilde{H}_{\perp x}$ be defined so as to reproduce the LTFP equations of (15).

More explicitly, we require $\tilde{H}_{\perp x}$ to be such that its action on $\rho_{\sigma}$ generates the LTFP equations

$$
\left\{\rho_{\sigma}[\chi], H_{\perp x}\right\}=\frac{\delta \rho_{\sigma}[\chi]}{\delta \xi^{\perp}}
$$


which translates to

$$
\frac{\tilde{\delta} \tilde{H}_{\perp x}}{\tilde{\delta} \Phi_{\sigma}[\chi]}=-\frac{1}{g_{x}^{1 / 2}} \frac{\delta}{\delta \chi_{x}}\left(\rho_{\sigma}[\chi] \frac{\delta \Phi_{\sigma}[\chi]}{\delta \chi_{x}}\right)
$$

It is simple to check that the $\tilde{H}_{\perp x}$ that satisfies this condition is given by

$$
\tilde{H}_{\perp}=\int D \chi \rho_{\sigma} \frac{1}{2 g_{x}^{1 / 2}}\left(\frac{\delta \Phi_{\sigma}}{\delta \chi_{x}}\right)^{2}+F_{x}\left[\rho ; g_{i j}\right]
$$

The first term in (64) is fixed by virtue of consistency with the LTFP equations, whereas $F_{x}\left[\rho ; g_{i j}\right]$ is a yet undetermined "constant" of integration, which may depend on $\rho$ as well as the metric. However, $F_{x}$ is not entirely arbitrary, its functional form is restricted by the Poisson bracket, Equation (59).

Before proceeding, note that, up until this point, our discussion of path independence has been developed on a formal level, largely independently of ED itself. Such a formalism on its own, however, is necessarily devoid of many crucial physical ingredients. For instance, in ED we define local time as a measure of the field fluctuations through (8), which leads to the LTFP equations. In principle, this has nothing to do with abstract parameters $\delta \xi_{x}^{A}$ introduced as part of local updating. It is only once we say that the entropic updating of ED must agree with the local time evolution generated by $\tilde{H}_{\perp x}$, made explicit in (13), that the two notions coincide. In ED, the clock that measures the local proper time $\delta \xi_{x}^{\perp}$ is nothing but the field fluctuations themselves.

Continuing with our task at hand, we seek a family of models that are consistent with Equation (59). This is accomplished for suitable choices of $F_{x}$. We pursue this in manner similar to [29]. Begin by rewriting $\tilde{H}_{\perp x}$ as

$$
\tilde{H}_{\perp x}=\tilde{H}_{\perp x}^{0}+F_{x},
$$

where we have introduced

$$
\tilde{H}_{\perp x}^{0}=\int D \chi \rho_{\sigma}\left(\frac{1}{2 g_{x}^{1 / 2}}\left(\frac{\delta \Phi_{\sigma}}{\delta \chi_{x}}\right)^{2}+\frac{g_{x}^{1 / 2}}{2} g^{i j} \partial_{i x} \chi_{x} \partial_{j x} \chi_{x}\right) .
$$

This amounts simply to a redefinition of the arbitrary $F_{x}$ in (64). An advantage of this definition, however, is that the newly defined $\tilde{H}_{\perp x}^{0}$ automatically satisfies

$$
\left\{\tilde{H}_{\perp x}^{0}, \tilde{H}_{\perp x^{\prime}}^{0}\right\}=\left(g_{x}^{i j} \tilde{H}_{j x}+g_{x^{\prime}}^{i j} \tilde{H}_{j x^{\prime}}\right) \partial_{i x} \delta\left(x, x^{\prime}\right) .
$$

Since $F_{x}\left[\rho ; g_{i j}\right]$ is independent of $\Phi$, we have that $\left\{F_{x}, F_{x^{\prime}}\right\}=0$, identically. Thus, finding a suitable $F_{x}$ amounts to satisfying

$$
\left\{\tilde{H}_{\perp x}^{0}, F_{x^{\prime}}\right\}=\left\{\tilde{H}_{\perp x^{\prime}}^{0}, F_{x}\right\}
$$

Clearly a necessary condition for an acceptable $F_{x}$ is that the Poisson bracket $\left\{\tilde{H}_{\perp x^{\prime}}^{0}, F_{x^{\prime}}\right\}$ must be symmetric upon exchange of $x$ and $x^{\prime}$. Since $\tilde{H}_{\perp x}^{0}$ must itself reproduce the LTFP equations, the condition $(68)$ translates to $[28,29]$

$$
\frac{1}{g_{x}^{1 / 2}} \frac{\delta}{\delta \chi_{x}}\left(\rho_{\sigma} \frac{\delta}{\delta \chi_{x}} \frac{\tilde{\delta} F_{x^{\prime}}}{\tilde{\delta} \rho_{\sigma}}\right)=\frac{1}{g_{x^{\prime}}^{1 / 2}} \frac{\delta}{\delta \chi_{x^{\prime}}}\left(\rho_{\sigma} \frac{\delta}{\delta \chi_{x^{\prime}}} \frac{\tilde{\delta} F_{x}}{\tilde{\delta} \rho_{\sigma}}\right)
$$

which is an equation linear in $F_{x}$. 
A complete description of solutions to Equation (69) lies outside the scope of the current work. However, a restricted family of solutions, which are nonetheless of physical interest, can be found for $F_{x} \mathrm{~s}$ of the form

$$
F_{x}[\rho]=\int D \chi f_{x}\left(\rho, \frac{\delta \rho}{\delta \chi_{x}} ; g_{i j x}\right),
$$

where $f_{x}$ is a function, not functional, of its arguments. For such a special type of $F_{x}$ one can check by substitution into (69) that

$$
f_{x} \sim g_{x}^{1 / 2} \rho \chi_{x}^{n} \quad(\text { integer } n) \text { and } f_{x} \sim \frac{\rho}{g_{x}^{1 / 2}}\left(\frac{\delta \log \rho}{\delta \chi_{x}}\right)^{2}
$$

are acceptable solutions. Since Equation (69) is linear in $F_{x}$, solutions can be superposed so that a suitable family of $\tilde{H}_{\perp x}$ s is given by

$$
\tilde{H}_{\perp x}=\int D \chi \rho_{\sigma}\left(\frac{1}{2 g_{x}^{1 / 2}}\left(\frac{\delta \Phi_{\sigma}}{\delta \chi_{x}}\right)^{2}+\frac{g_{x}^{1 / 2}}{2} g^{i j} \partial_{i x} \chi_{x} \partial_{j x} \chi_{x}+g_{x}^{1 / 2} V_{x}\left(\chi_{x}\right)+\frac{\lambda}{g_{x}^{1 / 2}}\left(\frac{\delta \log \rho_{\sigma}}{\delta \chi_{x}}\right)^{2}\right),
$$

where $V_{x}=\sum_{n} \lambda_{n} \chi_{x}^{n}$ is a function that is polynomial in $\chi_{x}$.

As discussed in [29], the last term can be interpreted as the "local quantum potential." To see this we recall that in flat space-time the quantum potential is given by [27]

$$
Q=\int d^{3} x \int D \chi \rho \lambda\left(\frac{\delta \log \rho}{\delta \chi_{x}}\right)^{2}
$$

The transition to a curved space-time is made by making the substitutions

$$
d^{3} x \rightarrow g_{x}^{1 / 2} d^{3} x \text { and } \frac{\delta}{\delta \chi_{x}} \rightarrow \frac{1}{g_{x}^{1 / 2}} \frac{\delta}{\delta \chi_{x}},
$$

yielding the result

$$
Q_{\sigma}=\int d^{3} x \int D \chi \rho \frac{\lambda}{g_{x}^{1 / 2}}\left(\frac{\delta \log \rho}{\delta \chi_{x}}\right)^{2} .
$$

The term inside the spatial integral is exactly what appears in (71), which justifies the name. The contribution of the local quantum potential to the energy is such that those states that are more smoothly spread out in configuration space tend to have lower energy. The corresponding coupling constant $\lambda>0$ controls the relative importance of the quantum potential; the case $\lambda<0$ is excluded because it leads to instabilities.

\subsubsection{The Gravitational Super-Hamiltonian}

We now proceed to determining the last remaining element of our scheme, the gravitational super-Hamiltonian $H_{\perp x}^{G}$. Fortunately, under the assumption of non-derivative coupling mentioned above, it is possible to completely separate the Poisson bracket relations (32)-(34) into pieces that are purely gravitational and those that are pure "matter." Consequently, to determine $H_{\perp x}^{G}$ it suffices merely to solve the Poisson bracket relations (56)-(58), which involve only the gravitational variables $g_{i j}$ and $\pi^{i j}$.

Such a task, however, is mathematically equivalent to determining the generators of pure geometrodynamics, in which the coupling to "matter" is absent. But it is precisely this latter challenge which was, in fact, addressed by the efforts of HKT in [44] - they proposed a solution to exactly those brackets that appear in (56)-(58). Their main result was an important one: the only time-reversible 
solution to Equations (56)-(58) is nothing less than Einstein's GR in vacuum. We briefly review their argument here, and adapt their solution to our current work in ED.

To begin, since $g_{i j}$ is the intrinsic metric of the surface, its behavior under a purely normal deformation is known [60] to be

$$
\delta g_{i j}(x)=£_{\delta \xi^{\perp}} g_{i j x}=-2 K_{i j x}
$$

where $K_{i j}(x)=K_{i j x}$ is a symmetric $(0,2)$ tensor that is called the extrinsic curvature. Note that identifying $K_{i j x}$ with the response of $g_{i j x}$ under a normal deformation is a geometric requirement, not a dynamical one; without this, we could not interpret $g_{i j}$ as residing on a space-like cut of space-time. That being said, $K_{i j x}$ is at this juncture an undetermined functional of the canonical variables, and more to the point, we do not assume at the moment any simple relationship with the momenta $\pi_{x}^{i j}$-this must be derived.

Alternatively, the deformation in (73) must also be attainable using the normal generator $H_{\perp x}^{G}$ :

$$
\delta g_{i j x}=\int d x^{\prime}\left\{g_{i j x}, H_{\perp x^{\prime}}^{G}\right\} \delta \xi_{x^{\prime}}^{\perp}
$$

More explicitly, $H_{\perp x}^{G}$ should satisfy

$$
\frac{\delta H_{\perp x^{\prime}}^{G}}{\delta \pi_{x}^{i j}}=-2 K_{i j x} \delta\left(x, x^{\prime}\right)
$$

The appearance of the Dirac delta in Equation (74) implies that $H_{\perp x}^{G}$ is local in $\pi_{x}^{i j}$. Therefore $H_{\perp x}^{G}$ is a function, not a functional, of $\pi_{x}^{i j}$.

Such a simplification turns out to be important: once $H_{\perp x}^{G}$ is a function of $\pi^{i j}$, it is possible to produce an ansatz for $H_{\perp x}^{G}$ in terms of powers of $\pi^{i j}$. HKT then supplement this with an additional simplifying assumption: that geometrodynamics be time-reversible. This has the advantage of removing all terms in $H_{\perp x}^{G}$ that have odd powers of $\pi_{x}^{i j}$.

Incorporating these ingredients, it is then possible to consider $H_{\perp x}^{G}$ s of the form

$$
H_{\perp x}^{G}=\sum_{n=0}^{\infty} G_{i_{1} j_{1} i_{2} j_{2} \cdots i_{2 n} j_{2 n} x}^{(2 n)} \pi_{x}^{i_{1} j_{1}} \pi_{x}^{i_{2} j_{2}} \cdots \pi_{x}^{i_{2 n} j_{2 n}}
$$

where the coefficients $G_{i j \ldots}^{(2 n)}$ are functionals of the metric $g_{i j}$, but depend on the point $x$. Furthermore, since $H_{\perp x}^{G}$ is scalar density and $\pi^{i j}$ a tensor density, $G_{i j \ldots}^{(2 n)}$ must transform as a tensor density of weight $1-2 n$. Since $\pi^{i j}$ is a symmetric tensor we expect $G_{i j \ldots .}^{(2 n)}$ to be symmetric under exchange of indices $i_{a} j_{a} \leftrightarrow j_{a} i_{a}$ for any pair $a$; also, $G_{i j \ldots}^{(2 n)}$ should be symmetric on interchange of any pair $i_{a} j_{a} \leftrightarrow i_{b} j_{b}$ as this just corresponds to exchanging the $\pi \mathrm{s}$.

Naturally, one narrows the allowable $H_{\perp x}^{G}$ by inserting the ansatz (75) into the Poisson bracket (56). Without delving too far into the details (which can be found in [44]) we quote their solution. Only the $n=0,1$ terms survive, giving

$$
H_{\perp x}^{G}=\kappa G_{i j k l} \pi^{i j} \pi^{k l}-\frac{g^{1 / 2}}{2 \kappa}(R-\Lambda),
$$

where we have introduce the super metric

$$
G_{i j k l}=\frac{1}{g_{x}^{1 / 2}}\left(g_{i k} g_{j l}+g_{i l} g_{j k}-g_{i j} g_{k l}\right)
$$


and where $R$ is the Ricci scalar for the metric $g_{i j}$.

The constant $\kappa$ is a coefficient that, eventually, determines the coupling to "matter." We follow standard convention in identifying it as $\kappa=8 \pi G$, where $G$ is Newton's constant. The other parameter $\Lambda$, of course, is the cosmological constant. For simplicity, going forward we set $\Lambda=0$.

Putting everything together, we have that $H_{\perp x}^{G}$ takes the form

$$
H_{\perp x}^{G}=\frac{\kappa}{g_{x}^{1 / 2}}\left(2 \pi^{i j} \pi_{i j}-\pi^{2}\right)-\frac{g^{1 / 2}}{2 \kappa} R
$$

where $\pi=\pi^{i j} g_{i j}=\operatorname{Tr}\left(\pi^{i j}\right)$. This is exactly the standard gravitational super-Hamiltonian obtained by Dirac [57] and ADM [58] by starting from the Einstein-Hilbert Lagrangian.

Total super-Hamiltonian

Putting together the ingredients of this section, the total super-Hamiltonian is thus

$$
H_{\perp x}=H_{\perp x}^{G}+\tilde{H}_{\perp x}
$$

where $H_{\perp x}^{G}$ is given above by Equation (78) and where a suitable family of $\tilde{H}_{\perp x}$ s has been identified in Equation (65). With this, the super-Hamiltonian constraint is then just

$$
H_{\perp x}=H_{\perp x}^{G}+\tilde{H}_{\perp x} \approx 0 .
$$

\section{The Dynamical Equations}

As argued by HKT, although the role of space-time was crucial to the developing the Equations (32)-(35), one notices that all signs of the enveloping space-time have dropped out in the closing relations (32)-(34). (For instance, they depend on the surface metric $g_{i j}$, but not on its extrinsic curvature $K_{i j}$ ). Thus in geometrodynamics we can dispense with the notion of an a priori given space-time and instead consider the three-dimensional Riemannian manifold $\sigma_{t}$ as primary.

The idea is a simple one. The canonical variables are evolved with the generators $H_{A x}$, satisfying Equations (32)-(35). Such an evolution will cause both the "matter" and geometry to change. We might then give this manifold with updated intrinsic geometry a new name, $\sigma_{t^{\prime}}$. Repeating this procedure results in an evolution of the dynamical variables, and consequently, what one might view as a succession of manifolds $\left\{\sigma_{t}\right\}$, parameterized by the label $t$. Thus this iterative process constructs a space-time, step by step. We now investigate the dynamical equations that result from this procedure.

\subsection{Some Formalism}

Consider the evolution of an arbitrary functional $T_{t}$ of the dynamical variables defined on $\sigma_{t}$,

$$
\delta T_{t}=T_{t+d t}-T_{t}=\int d x\left\{T_{t}, H_{A x}\right\} \delta \xi_{x}^{A}=\int d x N_{x t}^{A}\left\{T_{t}, H_{A x}\right\} d t,
$$

where we have introduced four arbitrary functions

$$
N_{x t}=\frac{\delta \xi_{x}^{\perp}}{d t} \quad \text { and } \quad N_{x t}^{i}=\frac{\delta \xi_{x}^{i}}{d t}
$$

which are the lapse and vector shift, respectively. Just as the evolution parameters $\delta \xi_{x}^{A}$ were completely arbitrary, these functions can be freely specified, and amount eventually to picking a particular foliation of space-time. At the moment, the lapse and shift have no definite geometrical meaning. But as is well known, we can eventually identify the lapse $N$ and shift $N^{i}$ as being related to components of 
the space-time metric ${ }^{4} g_{\mu v} \equiv \gamma_{\mu v}$. (We changed the symbol temporarily to avoid confusion). More specifically, we would have [44]

$$
N=\left(-\gamma^{00}\right)^{-1 / 2} \text { and } N_{i}=\gamma_{0 i}
$$

As in the Dirac approach to geometrodynamics, the $N_{x t}^{A}=\left(N_{x t}, N_{x t}^{i}\right)$ are not functionals of the canonical variables; therefore, we can rewrite Equation (81) as

$$
\delta T_{t}=\left\{T_{t}, H\left[N, N^{i}\right]\right\} d t,
$$

where we have introduced the notion of an "integrated", or "smeared" Hamiltonian

$$
H\left[N, N^{i}\right]=\int d x\left(N_{x t} H_{\perp x}+N_{x t}^{i} H_{i x}\right)
$$

that, for given $N_{x t}^{A}$, generates an evolution parameterized by $t$. This global $H\left[N, N^{i}\right]$ (note the spatial integral) conforms more naturally to our typical notions of a Hamiltonian. Thus, the derivative with respect to the parameter $t$ is

$$
\partial_{t} T_{t} \equiv \frac{\delta T_{t}}{d t}=\left\{T_{t}, H\left[N, N^{i}\right]\right\}
$$

or more explicitly by

$$
\partial_{t} T_{t}=\int d x\left(N_{x t}\left\{T_{t}, H_{\perp x}\right\}+N_{x t}^{i}\left\{T_{t}, H_{i x}\right\}\right)
$$

and more succinctly

$$
\partial_{t} T_{t}=£_{m} T_{t}+£_{N^{i}} T_{t}
$$

where the vector $m_{x}^{\mu}=N_{x t} n_{x}^{\mu}$ is the so-called evolution vector.

\subsection{The Evolution of the "Matter" Sector}

The goal is to determine the evolution of the probability distribution $\rho_{t}[\chi]$ and phase functional $\Phi_{t}[\chi]$, given an initial state $\left(\rho_{t}, \Phi_{t} ; g_{i j t}, \pi_{t}^{i j}\right)$ that satisfies the initial value constraints (35).

\subsubsection{Dynamical Equations for the Probability and Phase}

Given the basic dynamical law, Equation (85), the time evolutions of the variables $\rho_{t}$ and $\Phi_{t}$ are given by

$$
\partial_{t} \rho_{t}=\left\{\rho_{t}, H\right\} \quad \text { and } \quad \partial_{t} \Phi_{t}=\left\{\rho_{t}, H\right\},
$$

where $H$ is the smeared Hamiltonian given above. Thus, in accordance with Equation (86), we have the result

$$
\begin{aligned}
\partial_{t} \rho_{t} & =\int d x\left(N_{x t}\left\{\rho_{t}, H_{\perp x}\right\}+N_{x t}^{i}\left\{\rho_{t}[\chi], H_{i x}\right\}\right) \\
\partial_{t} \Phi_{t} & =\int d x\left(N_{x t}\left\{\Phi_{t}, H_{\perp x}\right\}+N_{x t}^{i}\left\{\Phi_{t}, H_{i x}\right\}\right) .
\end{aligned}
$$


Using the family of $H_{\perp x}$ s that we identified in Equation (79) and the super-momentum $H_{i x}$ in Equation (51) we can compute all of the necessary Poisson brackets. From the super momentum in Equation (51) we can compute the tangential pieces

$$
\begin{aligned}
\left\{\rho_{t}[\chi], H_{i x}\right\} & =\frac{\tilde{\delta} \tilde{H}_{i x}}{\tilde{\delta} \tilde{\Phi}_{t}}=\frac{\delta \rho_{t}}{\delta \chi_{x}} \partial_{i x} \chi_{x}, \\
\left\{\Phi_{t}[\chi], H_{i x}\right\} & =-\frac{\tilde{\delta} \tilde{H}_{i x}}{\tilde{\delta} \tilde{\rho}_{t}}=\frac{\delta \Phi_{t}}{\delta \chi_{x}} \partial_{i x} \chi_{x} .
\end{aligned}
$$

Of course, the entire family of $\tilde{H}_{\perp x} \mathrm{~s}$ was designed to reproduce the LTFP equations; thus, by construction we have

$$
\left\{\rho_{t}[\chi], H_{\perp x}\right\}=\frac{\tilde{\delta} \tilde{H}_{\perp x}}{\tilde{\delta} \tilde{\Phi}_{t}}=-\frac{1}{g_{x}^{1 / 2}} \frac{\delta}{\delta \chi_{x}}\left(\rho_{t} \frac{\delta \Phi_{t}}{\delta \chi_{x}}\right)
$$

in agreement with Equation (15). The remaining Poisson bracket determines the local time evolution of the phase functional $\Phi_{t}$ and is given by

$$
\left\{\Phi_{t}[\chi], H_{\perp x}\right\}=-\frac{\tilde{\delta} \tilde{H}_{\perp x}}{\tilde{\delta} \tilde{\rho}_{t}}=-\frac{1}{2 g_{x}^{1 / 2}}\left(\frac{\delta \Phi_{t}[\chi]}{\delta \chi_{x}}\right)^{2}+\frac{g_{x}^{1 / 2}}{2} g^{i j} \partial_{i} \chi_{x} \partial_{j} \chi_{x}+\frac{\tilde{\delta} F_{x}[\rho]}{\tilde{\delta} \rho_{t}[\chi]},
$$

where $F_{x}$ is of the form specified by Equation (65).

\subsubsection{The Local Time Hamilton-Jacobi Equations}

To interpret the local Equations (94), we write the full time evolution for the phase functional by inserting Equations (94) and (92) into Equation (90), yielding

$$
-\partial_{t} \Phi_{t}=\int d x\left[N_{x t}\left(\frac{1}{2 g_{x}^{1 / 2}}\left(\frac{\delta \Phi_{t}}{\delta \chi_{x}}\right)^{2}+\frac{g_{x}^{1 / 2}}{2} g^{i j} \partial_{i} \chi_{x} \partial_{j} \chi_{x}+\frac{\tilde{\delta} F_{x}}{\tilde{\delta} \rho_{t}}\right)+N_{x t}^{i} \frac{\delta \Phi_{t}}{\delta \chi_{x}} \partial_{i x} \chi_{x}\right] .
$$

To bring this equation into a more familiar form we consider the special case of flat space-time by setting the metric to be a Kroenecker delta $g_{i j}=\delta_{i j}$, so that $g_{x}^{1 / 2}=1$, and we let $N=1, N^{i}=0$. Moreover, for simplicity we also make the assignment $F_{x}=0$. This results in a time evolution for $\Phi_{t}$ that has the form

$$
-\partial_{t} \Phi_{t}=\int d x\left(\frac{1}{2}\left(\frac{\delta \Phi_{t}}{\delta \chi_{x}}\right)^{2}+\frac{1}{2} \delta^{i j} \partial_{i} \chi_{x} \partial_{j} \chi_{x}\right),
$$

which is exactly the classical Hamilton-Jacobi equation for a massless Klein-Gordon field in flat space-time. Thus, in analogy with the LTFP equations, we refer to Equation (94) as the local time Hamilton-Jacobi (LTHJ) equations, as there is one equation of the Hamilton-Jacobi type for every spatial point.

The LTFP and LTHJ equations, with the tangential Equation (92), and the evolution Equation (90), give us the ability to evolve an appropriately chosen initial state $\left(\rho_{t}, \Phi_{t}\right)$. In general, this is a coupled non-linear evolution driven by a dependence on the metric $g_{i j}$. To a large extent, this completes our discussion of how the epistemic variables evolve. In a subsequent section, however, we discuss in some detail the dynamics of a specific class of models, those that involve the local quantum potential.

\subsection{The Evolution of the Geometrical Variables}

We now review the content of the well-known Einstein's equations written within the canonical language. (A good review of these equations is given, for example, by ADM [59]). 


\subsubsection{Evolution of Metric}

The goal is to determine the evolution of the geometrical variables $\left(g_{i j}, \pi^{i j}\right)$. Beginning with the metric $g_{i j}$ defined on some initial three-space $\sigma_{t}$, we wish to determine how it evolves in response to the generators $H_{A x}$. Applying Equation (86) for the time derivative, we have that

$$
\partial_{t} g_{i j x}=\int d x^{\prime}\left\{g_{i j x}, H_{A x^{\prime}}\right\} N_{x^{\prime} t}^{A}=\int d x^{\prime} \frac{\delta H_{A x^{\prime}}}{\delta \pi_{x}^{i j}} N_{x^{\prime} t}^{A} .
$$

To compute this, recall that the tangential piece is known from Equations (37) and (42). This gives us

$$
£_{N^{i}} g_{i j x}=\int d x^{\prime} \frac{\delta H_{i x^{\prime}}}{\delta \pi_{x}^{i j}} N_{x^{\prime} t}^{i}=\nabla_{i} N_{j x t}+\nabla_{j} N_{i x t}
$$

where $\ell_{N^{i}} g_{i j x}$ is just the Lie derivative along the vector field defined by the shift $N^{i}$, and where $N_{i x t}=g_{i j x} N_{x t}^{j}$.

To obtain the remaining piece, first differentiate the $H_{\perp x}$ given in Equation (78)

$$
\frac{\delta H_{\perp x^{\prime}}}{\delta \pi_{x}^{i j}}=\frac{4 \kappa}{g_{x}^{1 / 2}}\left(\pi_{i j x}-\frac{1}{2} \pi_{x} g_{i j x}\right) \delta\left(x, x^{\prime}\right)
$$

where $\pi_{i j}$ is the conjugate momentum with its indices lowered, and $\pi=g_{i j} \pi^{i j}=\operatorname{Tr}\left(\pi^{i j}\right)$ is the trace of the gravitational momentum. Putting these terms together, Equation (96) reads as

$$
\begin{aligned}
\partial_{t} g_{i j x} & =£_{m} g_{i j x}+£_{N^{i}} g_{i j x} \\
£_{m} g_{i j x} & =\frac{2 \kappa}{g_{x}^{1 / 2}}\left(2 \pi_{i j x}-\pi_{x} g_{i j x}\right) N_{x t} \\
£_{N^{i}} g_{i j x} & =\nabla_{i} N_{j x t}+\nabla_{j} N_{i x t} .
\end{aligned}
$$

This gives us the evolution of the metric with foliation parameter $t$.

We can also now identify the extrinsic curvature tensor

$$
K_{i j}=\frac{\kappa}{g_{x}^{1 / 2}}\left(\pi_{x} g_{i j x}-2 \pi_{i j x}\right) .
$$

Inverting this relationship, we get $\pi^{i j}$ in terms of $K^{i j}$, which yields

$$
\pi_{x}^{i j}=\frac{g_{x}^{1 / 2}}{2 \kappa}\left(K g_{x}^{i j}-K_{x}^{i j}\right)
$$

where $K=K_{i j} g^{i j}=\operatorname{Tr}\left(K_{i j}\right)$ is the trace of $K_{i j}$. This is of some interest if one wishes to compare the canonical formulation to the standard so-called "Lagrangian" approach (see, e.g., [65]).

\subsubsection{Evolution of Conjugate Momentum}

To this point, the dynamics of the geometry have not differed from purely classical geometrodynamics, such as those of HKT. This is because the "matter" super-Hamiltonian and super-momentum were, by definition, completely independent of $\pi^{i j}$ (due, of course, to the non-derivative coupling assumption); therefore, the evolution of $g_{i j}$ did not receive contributions from the "matter" sector. This changes when we consider the dynamics of the conjugate momentum $\pi^{i j}$.

The evolution of $\pi^{i j}$ is determined via the equation

$$
\partial_{t} \pi_{x}^{i j}=\int d x^{\prime}\left\{\pi_{x}^{i j}, H_{A x^{\prime}}\right\} N_{x^{\prime} t}^{A}=-\int d x^{\prime} \frac{\delta H_{A x^{\prime}}}{\delta g_{i j x}} N_{x^{\prime} t}^{A} .
$$


Recalling now that both the gravitational and "matter" super-Hamiltonians $H_{\perp x}=H_{\perp x}^{G}\left[g_{i j}, \pi^{i j}\right]+$ $\tilde{H}_{\perp x}\left[\rho, \Phi ; g_{i j}\right]$ depend explicitly on the metric, but that $\tilde{H}_{i x}$ does not, this expression slightly simplifies to

$$
\partial_{t} \pi_{x}^{i j}=-\int d x^{\prime}\left(N_{x^{\prime}} \frac{\delta H_{\perp x^{\prime}}^{G}}{\delta g_{i j x}}+N_{x^{\prime} t} \frac{\delta \tilde{H}_{\perp x^{\prime}}}{\delta g_{i j x}}+N_{x^{\prime} t}^{i} \frac{\delta H_{i x^{\prime}}^{G}}{\delta g_{i j x}}\right),
$$

where we have have separated the contributions from the gravitational and "matter" sectors. In the notation of Equation (87) we write this as

$$
\partial_{t} \pi_{x}^{i j}=£_{m} \pi_{x}^{i j}+£_{N^{i}} \pi_{x}^{i j}
$$

where

$$
£_{m} \pi_{x}^{i j}=£_{m}^{G} \pi_{x}^{i j}+£_{m}^{M} \pi_{x}^{i j}
$$

and

$$
\begin{aligned}
£_{m}^{G} \pi_{x}^{i j} & =-\int d x^{\prime}\left(N_{x^{\prime} t} \frac{\delta H_{\perp x^{\prime}}^{G}}{\delta g_{i j x}}\right), \\
£_{m}^{M} \pi_{x}^{i j} & =-\int d x^{\prime}\left(N_{x^{\prime} t} \frac{\delta \tilde{H}_{\perp x^{\prime}}}{\delta g_{i j x}}\right), \\
£_{N^{i}} \pi_{x}^{i j} & =-\int d x^{\prime}\left(N_{x^{\prime} t}^{i} \frac{\delta H_{i x^{\prime}}^{G}}{\delta g_{i j x}}\right) .
\end{aligned}
$$

From (43) the last term is fairly easy to determine.

$$
£_{N^{i}} \pi_{x}^{i j}=\nabla_{k x}\left(\pi_{x}^{i j} N_{x}^{k}\right)-\pi_{x}^{i k} \nabla_{k x} N_{x}^{j}-\pi_{x}^{k j} \nabla_{k x} N_{x}^{i}
$$

The calculation of the other two terms is much more involved. Fortunately, the expression for $£_{m}^{G} \pi^{i j}$ is already well known [59,60], and we merely quote the result:

$$
\begin{aligned}
E_{m}^{G} \pi_{x}^{i j} & =-\frac{g^{1 / 2}}{2 \kappa}\left(R_{x}^{i j}-\frac{1}{2} g_{x}^{i j} R_{x}\right) N_{x t}+\frac{\kappa}{g_{x}^{1 / 2}} g_{x}^{i j}\left(\pi_{x}^{k l} \pi_{k l x}-\frac{1}{2} \pi_{x}^{2}\right) N_{x t} \\
& -\frac{4 \kappa}{g_{x}^{1 / 2}}\left(\pi_{x}^{i k} \pi_{k x}^{j}-\frac{1}{2} \pi_{x} \pi_{x}^{i j}\right) N_{x t}+\frac{g_{x}^{1 / 2}}{2 \kappa}\left(\nabla_{x}^{i} \nabla_{x}^{j} N_{x t}-g_{x}^{i j} \nabla_{x}^{k} \nabla_{k x} N_{x t}\right) .
\end{aligned}
$$

To calculate the remaining piece $£_{m}^{M} \pi_{x}^{i j}$ we first recall that having assumed a non-derivative coupling of gravity to matter, the metric $g_{i j}$ appears in $\tilde{H}_{\perp x}$ as an undifferentiated function (not a functional) and without any derivatives [47], which implies that

$$
\frac{\delta \tilde{H}_{\perp x^{\prime}}}{\delta g_{i j x}}=\frac{\partial \tilde{H}_{\perp x}}{\partial g_{i j x}} \delta\left(x, x^{\prime}\right),
$$

obtaining

$$
£_{m}^{M} \pi_{x}^{i j}=-\frac{\partial \tilde{H}_{\perp x}}{\partial g_{i j x}} N_{x t}
$$


Using Equation (65), for an arbitrary choice of $F_{x}$, but one that still satisfies Equation (59), the "matter" source has the form

$$
£_{m}^{M} \pi_{x}^{i j}=N_{x t}\left(\frac{\partial \tilde{H}_{\perp x}^{0}}{\partial g_{i j x}}+\frac{\partial F_{x}}{\partial g_{i j x}}\right)
$$

with

$$
\frac{\partial \tilde{H}_{\perp x}^{0}}{\partial g_{i j x}}=-\frac{1}{2} \int D \chi \rho\left[\left(\frac{1}{g_{x}^{1 / 2}}\left(\frac{\delta \Phi}{\delta \chi_{x}}\right)^{2}-g_{x}^{1 / 2} g_{x}^{k l} \partial_{k x} \chi_{x} \partial_{l x} \chi_{x}\right) g_{x}^{i j}-g_{x}^{1 / 2} \partial_{x}^{i} \chi_{x} \partial_{x}^{j} \chi_{x}\right],
$$

where $\tilde{H}_{\perp x}^{0}$ was given in Equation (66), and where $\partial_{x}^{i} \chi_{x}=g_{x}^{i j} \partial_{j x} \chi_{x}$.

In total, the equations of motion for the gravitational field follow Hamilton's equations for the gravitational variables $\left(g_{i j x}, \pi_{x}^{i j}\right)$, given by Equation (98) for the evolution of the metric, and for the conjugate momentum $\pi_{x}^{i j}$ we have

$$
\partial_{t} \pi_{x}^{i j}-£_{n}^{G} \pi_{x}^{i j}-€_{N^{i}} \pi_{x}^{i j}=-N_{x t} \frac{\partial \tilde{H}_{\perp x}}{\partial g_{i j x}} .
$$

This is an equation in which geometrical variables on the left-hand side are sourced by the variables $(\rho, \Phi)$, which contain all the information available about the field $\chi_{x}$ on the right-hand side. Below we will discuss this equation in the presence of "quantum matter."

\section{Quantum Sources of Gravitation}

The transition to what may be termed a quantum form of dynamics amounts to an appropriate choice of the functional $F_{x}\left[\rho ; g_{i j}\right]$ (see, e.g., [27-29]). In particular, for $F_{x}\left[\rho ; g_{i j}\right]$ we choose exactly the local quantum potential introduced as part of (71). A convenient choice for the coupling constant is $\lambda=1 / 8$, which implies that we work in a system of units where $\hbar=c=1$. (As argued, for instance, in [22], there is no loss of generality in making this choice. For the case of nonrelativistic particles it can be proven [23] that an ED that preserves the appropriate symplectic and metric structures implies the presence of a quantum potential with the correct coefficient).

The connection to conventional quantum theory is made explicit by a change of variables from the probability $\rho$ and phase $\Phi$ to the complex variables

$$
\Psi=\rho^{1 / 2} e^{i \Phi} \quad \text { and } \quad \Psi^{*}=\rho^{1 / 2} e^{-i \Phi} .
$$

Such a change of variables is, in fact, a canonical transformation, and so, the new variables form a canonical pair given by $\left(\Psi, i \Psi^{*}\right)$, which obey a natural generalization of the standard Poisson bracket relations

$$
\left\{\Psi[\chi], i \Psi^{*}\left[\chi^{\prime}\right]\right\}=\delta\left[\chi-\chi^{\prime}\right],
$$

where $\delta\left[\chi-\chi^{\prime}\right]$ is a Dirac delta functional.

\subsection{Quantum Operators in ED}

Having chosen an $F_{x}\left[\rho ; g_{i j}\right]$ of the type described above, the ensemble generators $\tilde{H}_{A x}$ take a particularly special form

$$
\tilde{H}_{A x}=\int D \chi \Psi^{*} \hat{H}_{A x} \Psi=\left\langle\hat{H}_{A x}\right\rangle
$$


which can be viewed as both the expected value with respect to the probability $\rho$ of a Hamiltonian density, as in (71), and the expectation value of the local Hamiltonian operators

$$
\begin{aligned}
\hat{H}_{\perp x} & =-\frac{1}{2 g^{1 / 2}} \frac{\delta^{2}}{\delta \chi_{x}^{2}}+\frac{g^{1 / 2}}{2} g^{i j} \partial_{i} \chi_{x} \partial_{j} \chi_{x}+g^{1 / 2} V_{x}\left(\chi_{x} ; g_{i j}\right) \\
\hat{H}_{i x} & =i \partial_{i} \chi_{x} \frac{\delta}{\delta \chi_{x}},
\end{aligned}
$$

with respect to the quantum state $\Psi$. To obtain the "matter" contribution in Equation (114) for the conjugate momentum $\pi_{x}^{i j}$, note that the metric appears in $\tilde{H}_{\perp x}$ through the density $g_{x}^{1 / 2}$ and the inverse metric $g_{x}^{i j}$. Variations of these quantities with respect to the metric $g_{i j x}$ are given by [65]

$$
\delta g_{x}=g g_{x}^{i j} \delta g_{i j x} \quad \text { and } \quad \delta g_{x}^{i j}=g^{i k} g^{j l} \delta g_{k l}
$$

Then

$$
\frac{\partial \tilde{H}_{\perp x}}{\partial g_{i j x}}=\int D \chi \Psi^{*} \frac{\partial \hat{H}_{\perp x}}{\partial g_{i j x}} \Psi
$$

where we have defined the operator

$$
\frac{\partial \hat{H}_{\perp x}}{\partial g_{i j x}}=\partial_{x}^{i} \chi_{x} \partial_{x}^{j} \chi_{x}+g^{i j}\left(\frac{1}{2 g_{x}^{1 / 2}} \frac{\delta^{2}}{\delta \chi_{x}^{2}}+\frac{g_{x}^{1 / 2}}{2} g^{k l} \partial_{k} \chi_{x} \partial_{l} \chi_{x}+V_{x}\left(\chi_{x}\right)\right)
$$

\subsection{Geometrodynamics with Quantum Sources}

Our goal here is to rewrite the main equations of geometrodynamics with sources given by quantum matter. We begin first with the constraint equations. With the aid of the local operators introduced in (118) and (119), the total Hamiltonian generators take the explicit form

$$
\begin{aligned}
H_{\perp x} & =\frac{\kappa}{g_{x}^{1 / 2}}\left(2 \pi_{x}^{i j} \pi_{i j x}-\pi_{x}^{2}\right)-\frac{g_{x}^{1 / 2}}{2 \kappa} R_{x}+\int D \chi \Psi^{*} \hat{H}_{\perp x} \Psi \\
H_{i x} & =-2 \nabla_{k x}\left(\pi_{x}^{k j} g_{i j x}\right)+\int D \chi \Psi^{*} \hat{H}_{i x} \Psi
\end{aligned}
$$

which are, of course, subject to the constraints

$$
H_{\perp x} \approx 0 \text { and } H_{i x} \approx 0
$$

As relations (121) and (122) coupled together with (123) make abundantly clear, the quantum state and the geometrical variables can no longer be treated as independent. This has important consequences for the time evolution of $\Psi$.

Moving on, note that the dynamical equation for the metric, given in Equation (98), does not depend directly on the choice of $F_{x}$, and therefore remains unchanged in the quantum context. The dynamical equation for the conjugate momentum $\pi_{x}^{i j}$, however, is modified. Using the operator introduced in (120), this becomes

$$
\partial_{t} \pi_{x}^{i j}-£_{n}^{G} \pi_{x}^{i j}-€_{N^{i}} \pi_{x}^{i j}=-N_{x t} \int D \chi \Psi^{*} \frac{\partial \hat{H}_{\perp x}}{\partial g_{i j x}} \Psi
$$

where $£_{m}^{G} \pi_{x}^{i j}=$ and $£_{N^{i}} \pi_{x}^{i j}$ are given in Equations (107) and (110), respectively. This is the crucial equation in which the dynamical geometry is itself affected by the epistemic state $\Psi$. 
Putting it all together, the Equations (121)-(124), and Equation (98) for the metric, constitute a system of equations that is formally equivalent to the SCEE put in the canonical form. However, that is where the similarities end. On several key issues of interpretation, in particular, the ED approach is vastly different from the SCEE as they are normally understood. Far from being trivial, these distinctions turn out to be quite important, since many objections (see, e.g., [66,67]) to the usual SCEE are, in fact, based on such considerations.

\subsection{Quantum Dynamics}

One advantage of the complex variables $\left(\Psi, \Psi^{*}\right)$ is that the dynamics take a familiar form. Indeed, since $\Psi$ and $\Psi^{*}$ are just functions of our canonical variables $(\rho, \Phi)$ we can just use Equation (87) to determine their evolution along a time parameter $t$, which gives

$$
\partial_{t} \Psi_{t}[\chi]=\int d x\left\{\Psi_{t}[\chi], H_{A x}\right\} N_{x t}^{A} .
$$

The tangential component is obtained in a straightforward fashion by

$$
\left\{\Psi_{t}[\chi], H_{i x}\right\}=\partial_{i} \chi_{x} \frac{\delta \Psi_{t}[\chi]}{\delta \chi_{x}},
$$

which is reasonable since this is just the Lie derivative of $\Psi[\chi]$ along the surface (compare, for example, to Equations (48) and (49) for $\rho[\chi]$ and $\Phi[\chi]$ ). The local normal evolution of $\Psi$, on the other hand, is given by

$$
\left\{\Psi_{t}[\chi], \tilde{H}_{\perp x}\right\}=-i \hat{H}_{\perp x} \Psi_{t}[\chi] .
$$

Inserting these results into Equation (125) for a general evolution of $\Psi_{t}[\chi]$, we then have

$$
i \partial_{t} \Psi_{t}[\chi]=\int d x\left(N_{x t} \hat{H}_{\perp x}+N_{x t}^{i} \hat{H}_{i x}\right) \Psi_{t}[\chi]
$$

Finally, substituting Equations (118) and (119) in for $\hat{H}_{\perp x}$ and $\hat{H}_{i x}$, respectively, yields the equation

$$
i \hbar \partial_{t} \Psi_{t}=\int d x\left[N_{x t}\left(-\frac{\hbar^{2}}{2 g^{1 / 2}} \frac{\delta^{2}}{\delta \chi_{x}^{2}}+\frac{c^{2}}{2} g_{x}^{1 / 2} g^{i j} \partial_{i} \chi_{x} \partial_{j} \chi_{x}+g^{1 / 2} V_{x}\right)-\hbar c N_{x t}^{i} \partial_{i} \chi_{x} \frac{\delta}{\delta \chi_{x}}\right] \Psi_{t},
$$

where we have reinstated the constants $\hbar$ and $c$, as appropriate. Here Equation (129) is ostensibly just a linear equation for the complex variable $\Psi_{t}$, which suggests calling it a Schrödinger functional equation. We discuss below.

\section{But is it Quantum?}

The coupling to classical gravity described above implies violations of the superposition principle. To see this, we note that geometrodynamics are a constrained dynamical system, where the operative equations are given by the Hamiltonian constraints in (123). Solving these constraints often involves determining the components of the metric in terms of the quantum sources, which then gets fed back into evolution equations for $\Psi$. (This dynamic can most readily be seen within the linearized gravity regime, or the weak field limit. In fact, there are some arguments (see, e.g., [68]) that the so-called Newton-Schrödinger equation, which is a non-linear equation, results from the Newtonian limit of the semi-classical Einstein equations). This feedback leads to a non-linear time evolution (see, e.g., [66]) in which the $\Psi$ itself appears as a potential in Equation (129).

A natural question that arises is whether such dynamics can rightfully be called "quantum" or not. But this line of inquiry is rather misguided because the search for QG is, in many respects, the search for which criteria, in fact, constitute a quantum theory in the first place. Since the ED model formulated 
here involves the presence of $\hbar$, a wave equation for a complex wave function, an uncertainty principle, and non-local correlations, and limits itself to the standard quantum formalism in a flat space-time, we claim that it is these ingredients that are fundamental for defining a quantum theory. Additional features, such as the superposition principle, emerge here only as an effect confined to a limiting regime.

\section{Concluding Remarks}

The ED developed here couples quantum "matter" to classical gravity on the basis of three key principles: (1) A properly entropic setting wherein the dynamics of probability are driven by information encoded into constraints. (2) The preservation of a symplectic structure as a primary criterion for updating the evolving constraints. (3) Foliation invariance symmetry, enacted by representing the DHKT "algebra" in terms of the relevant Poisson brackets.

Our approach results in several interesting features. Although written in the relatively less common language of geometrodynamics, the Equations (98), (123) and (124) are formally equivalent to the so-called semi-classical Einstein equations (SCEE)

$$
G_{\mu \nu}=8 \pi G\left\langle\hat{T}_{\mu v}\right\rangle
$$

with classical Einstein tensor $G_{\mu \nu}$ (see, e.g., [65]), but sourced by the expected value of the quantum stress-energy tensor. (The components of the quantum stress-energy tensor are related in a simple manner to the ensemble quantities in Equations (117) and (120). The derivation of the SCEE from an action by Kibble and Randjbar-Daemi in [66] makes this relationship more precise). Such a theory of gravity has long been seen as a desirable step intermediate to a full theory of QG, in part because it contains well-established physics-QFTCS and classical GR-in the limiting cases where they are valid. But there has been much debate (see, e.g., [67,69-71]), on the other hand, as to the status of semi-classical theories as true QG candidate, with many harboring a negative view.

Here we do not propose a definitive rebuttal to those critics, but note that the ED formulation of SCEE has certain features that allow it to evade the most cogent criticisms. For one, a problem that is often raised against the SCEE is that it is proposed in a rather ad hoc manner, based on heuristic arguments. Indeed, the usual argument for the expected value on the right hand side of Equation (130) is at best a guess. In ED, on the other hand, the coupling of geometry to the expected value of quantum operators-made explicit in Equations (121)-(124) - is derived on the basis of well-defined assumptions and constraints. In other words, we derive the SCEE in ED from first principles. Indeed, these principles have already been tested elsewhere: not only do they provide a reconstruction of GR through the work of DHKT, but they also provide a reconstruction of both nonrelativistic quantum mechanics and relativistic quantum field theory. Furthermore, while there is no guarantee that such constraints and assumptions are adequate for a full QG theory, ED provides a framework wherein additional information can be incorporated as needed.

One source of confusion with the SCEE is the issue of what happens when the location of a macroscopic source is uncertain. (See e.g., $[67,68])$. Consider, for example, a macroscopic mass $m$ that is equally likely to be at $x_{1}$ or at $x_{2}$. Will the gravitational field itself be equally likely to point either towards $x_{1}$ or towards $x_{2}$ ? Or, will the gravitational field be as if generated by a mass $m$ located at the expected position $\left(x_{1}+x_{2}\right) / 2$ ? The ED resolution of this paradox shows the advantage of having a derivation from first principles. The $\left\langle\hat{T}_{\mu v}\right\rangle$ on the right of the SCEE is not the expectation value taken over any arbitrary source of uncertainty. The $\left\langle\hat{T}_{\mu \nu}\right\rangle$ was derived, or better, it was inferred from a very specific type of information that leads, by an abuse of language, to what one might call a pure quantum state. The issue then is what is the gravitational field generated by a pure state that happens to be a macroscopic "Schrödinger cat"? To the extent that this is a "pure" state then ED gives a sharp prediction: the gravitational field is generated by an $\left\langle\hat{T}_{\mu \nu}\right\rangle$ centered at the average position. But such a Schrödinger's cat state cannot be physically realized: it would immediately suffer decoherence. To analyze such a situation, the ED framework would need to be extended to incorporate information 
(i.e., additional constraints) that describes additional sources of uncertainty. In this extended ED it is conceivable that the gravitational field itself would be uncertain. So the conclusion is that there is no paradox; the two different predictions correspond to two different inferences arising from two different pieces of information.

Another family of objections revolve around paradoxes related to the problem of quantum measurement. As pointed out long ago by Kibble [72], these criticisms are premature as long as the interpretation of quantum mechanics is problematic and the problem of the collapse of the wave function remains unsolved. The issue is whether a measurement that induces a collapse of the wave function would result in a discontinuous change in the expected $\left\langle\hat{T}_{\mu v}\right\rangle$ with its attendant violations of causality.

Within the ED approach such objections do not arise. ED solves the problem of interpretation by starting with a clear definition of the ontology, which eliminates the interpretation problem. Furthermore, by including in its very foundation the epistemic tools for inference, ED also solves the problem of measurement [24,25]. In ED a measurement device is not described as a black box subject to rules that violate the Schrödinger equation but as a physical process subject to the very same laws that describe the rest of the world. As a result, in a fully covariant ED such as the model developed in this paper the process of measurement is described by the same causal flow of probability that characterizes any other physical process.

Yet another argument that has been raised against the SCEE is that the left hand side, featuring the gravitational field, is a "physical" ontic field, while the right hand side contains the quantum state $\Psi$, which is epistemic. Within the ED approach, however, the fact that $\left\langle\hat{T}_{\mu \nu}\right\rangle$ on the right hand side of (130) is epistemic necessarily forces the left hand side, the geometry, to be epistemic too. Indeed, a specific proposal along these lines has been offered in $[10,11]$, suggesting that the geometry of space-time itself can be given an entropic underpinning.

Finally, the Schrödinger equation derived here is quite unorthodox in that the dynamics of $\Psi$ follow a non-linear equation. This is quite problematic in the standard view of QT, where linearity is held as sacrosanct. But, as mentioned in the introduction, in the ED approach to quantum theory Hilbert spaces are not fundamental; they are introduced as a convenient trick precisely because of the calculational advantage of the linearity they induce. In the model developed here, such a trick cannot be carried out, and the superposition principle becomes the first casualty in a successful coupling of quantum matter with dynamical gravity.

The nonlinearity of the SCEEs does extreme violence to our understanding of quantum theory and raises many questions. Is the introduction of Hilbert spaces at all justified? Are density matrices at all useful? Can we expect something like the evolution from pure to mixed states? Or, in what seems more likely, are the very concepts of pure and mixed states so linked to the concept of Hilbert spaces that, absent the latter, the more useful description is just in terms of probabilities $\rho$ and phase fields $\Phi$ ? What is the generalization of von Neumann's entropy for such states? Can non-orthogonal states be distinguished? Can quantum states be cloned? The point of even raising such questions is precisely to emphasize the very different research directions one is led to once the standard tools of quantum mechanics are no longer fundamental and/or available. We must be open to the possibility that the proper way to model gravity might be as neither a quantum nor a classical theory but something else altogether-perhaps an inferential model based on information geometry in the spirit of the ED approach to QM.

Author Contributions: Both authors contributed to conceptualization, methodology, and typesetting of manuscript. All authors have read and agreed to the published version of the manuscript.

Funding: This research received no external funding.

Acknowledgments: We would like to thank M. Abedi, D. Bartolomeo, N. Carrara, N. Caticha, F. Costa, S. DiFranzo, K. Knuth, S. Nawaz, P. Pessoa, and K. Vanslette for many valuable discussions on entropy, inference, quantum theory, and much more. We would also like to thank the physics department of University at Albany-SUNY for their continued support. 
Conflicts of Interest: The authors declare no conflict of interest.

\section{References}

1. Carney, D.; Stamp, P.C.E.; Taylor, J.M. Tabletop experiments for quantum gravity: A user's manual. Class. Quantum Gravity 2019, 36, 034001. [CrossRef]

2. Surya, S. The causal set approach to quantum gravity. Living Rev. Relativ. 2019, 22, 5. [CrossRef]

3. Loll, R. Quantum gravity from causal dynamical triangulations: A review. Class. Quantum Gravity 2019, 37, 013002. [CrossRef]

4. Mukhi, S. String theory: A perspective over the last 25 years. Class. Quantum Gravity 2011, $28,153001$. [CrossRef]

5. Rovelli, C. Loop quantum gravity: The first 25 years. Class. Quantum Gravity 2011, 28, 153002. [CrossRef]

6. Henneaux, M.; Teitelboim, C. Quantization of Gauge Systems; Princeton University Press: Princeton, NJ, USA, 1994.

7. Jacobson, T. Thermodynamics of spacetime: The Einstein equation of state. Phys. Rev. Lett. 1995, 75, 1260. [CrossRef] [PubMed]

8. Verlinde, E.P. On the Origin of Gravity and the Laws of Newton. J. High Energy Phys. 2011, 2011. [CrossRef]

9. Verlinde, E.P. Emergent gravity and the dark universe. arXiv 2017, arXiv:1611.02269.

10. Caticha, A. Geometry from information geometry. In Proceedings of the 35th International Workshop on Bayesian Inference and Maximum Entropy Methods in Science and Engineering, Potsdam, NY, USA, 19-24 July 2015; Giffin, A., Knuth, K.H., Eds.; Volume 1757.

11. Caticha, A. The information geometry of space-time. In Proceedings of the 39th International Workshop on Bayesian Inference and Maximum Entropy Methods in Science and Engineering, Garching, Germany, 30 June-5 July 2019; von Toussaint, U., Preuss, R., Eds.; Volume 33.

12. Hall, M.J.W. Exact uncertainty approach in quantum mechanics and quantum gravity. Gen. Relativ. Gravit. 2005, 37, 1505-1515. [CrossRef]

13. Reginatto, M. Exact uncertainty principle and quantization: Implications for the gravitational field. Braz. J. Phys. 2005, 35, 476-480. [CrossRef]

14. Hardy, L. Towards quantum gravity: A framework for probabilistic theories with non-fixed causal structure. J. Phys. A 2007, 40, 3081. [CrossRef]

15. Hardy, L. The Construction Interpretation: Conceptual Roads to Quantum Gravity. arXiv 2018, arXiv:1807.10980.

16. Döring, A.; Isham, C.J. A topos foundation for theories of physics: I. Formal languages for physics. J. Math. Phys. 2008, 49, 053515. [CrossRef]

17. Döring, A.; Isham, C.J. A topos foundation for theories of physics: II. Daseinisation and the liberation of quantum theory. J. Math. Phys. 2008, 49, 053516. [CrossRef]

18. Caticha, A. Entropic Physics: Probability, Entropy, and the Foundations of Physics. Available online: https: / / www.albany.edu/physics/faculty/ariel-caticha (accessed on 12 July 2020).

19. Rosenkrantz, R.D. (Ed.) ET Jaynes: Papers on Probability, Statistics and Statistical Physics, 1st ed.; Springer Science and Business Media: Berlin/Heidelberg, Germany, 2012.

20. Caticha, A. From entropic dynamics to quantum theory. In Proceedings of the 29th International Workshop on Bayesian Inference and Maximum Entropy Methods in Science and Engineering, Oxford, MS, USA, 5-10 July 2009; Goggans, P.M., Chan, C.Y., Eds.; Volume 1193.

21. Caticha, A. Entropic dynamics, time, and quantum theory. J. Phys. A 2011, 44. [CrossRef]

22. Caticha, A.; Bartolomeo, D.; Reginatto, M. Entropic Dynamics: From entropy and information geometry to Hamiltonians and quantum mechanics. In Proceedings of the 34th International Workshop on Bayesian Inference and Maximum Entropy Methods in Science and Engineering, Amboise, France, 21-26 September 2014; Mohammad-Djafari, A., Barbaresco, F., Eds.; Volume 1641.

23. Caticha, A. The Entropic Dynamics approach to Quantum Mechanics. Entropy 2019, 21, 943. [CrossRef]

24. Johnson, D.T.; Caticha, A. Entropic dynamics and the quantum measurement problem. In Proceedings of the 31st International Workshop on Bayesian Inference and Maximum Entropy Methods in Science and Engineering, Waterloo, ON, Canada, 9-16 July 2011; Goyal, P., Giffin, A., Knuth, K. H., Vrscay, E., Eds.; Volume 1443. 
25. Vanslette, K.; Caticha, A. Quantum measurement and weak values in entropic dynamics. In Proceedings of the 36th International Workshop on Bayesian Inference and Maximum Entropy Methods in Science and Engineering, Ghent, Belgium, 10-15 July 2016; Verdoolaege, G., Ed.; Volume 1853.

26. Caticha, A. The entropic dynamics of relativistic quantum fields. In Proceedings of the 32nd International Workshop on Bayesian Inference and Maximum Entropy Methods in Science and Engineering, Garching, Germany, 15-20 July 2012; von Toussaint, U., Ed.; Volume 1553.

27. Caticha, A.; Ipek, S. Entropic Quantization of Relativistic Scalar Fields. In Proceedings of the 34th International Workshop on Bayesian Inference and Maximum Entropy Methods in Science and Engineering, Amboise, France, 21-26 September 2014; Mohammad-Djafari, A., Barbaresco, F., Eds.; Volume 1641.

28. Ipek, S.; Abedi, M.; Caticha, A. A covariant approach to entropic dynamics. In Proceedings of the 36th International Workshop on Bayesian Inference and Maximum Entropy Methods in Science and Engineering, Ghent, Belgium, 10-15 July 2016; Verdoolaege, G., Ed.; Volume 1853.

29. Ipek, S.; Abedi, M.; Caticha, A. Entropic dynamics: Reconstructing quantum field theory in curved space-time. Class. Quantum Gravity 2019, 36, 205013. [CrossRef]

30. Ipek, S.; Caticha, A. An Entropic Dynamics Approach to Geometrodynamics. In Proceedings of the 39th International Workshop on Bayesian Inference and Maximum Entropy Methods in Science and Engineering, Garching, Germany, 30 June-5 July 2019; von Toussaint, U., Preuss, R., Eds.; Volume 33.

31. Cox, R.T. Probability, frequency and reasonable expectation. Am. J. Phys. 1946, 14, 1-13. [CrossRef]

32. Wigner, E.P. Symmetries and Reflections: Scientific Essays; Indiana University Press: Bloomington, Indiana, 1967.

33. Jeffreys, H. An invariant form for the prior probability in estimation problems. Proc. R. Soc. Lond. A 1946, 186, 453-461.

34. Jaynes, E.T. Prior probabilities. Syst. Sci. Control. Eng. 1968, 4, 227-241. [CrossRef]

35. Nelson, E. Connection between Brownian motion and quantum mechanics. In Einstein Symposium Berlin; Springer: Berlin/Heidelberg, Germany, 1979; pp. 168-179.

36. Kibble, T.W.B. Geometrization of quantum mechanics. Commun. Math. Phys. 1979, 65, 189-201. [CrossRef]

37. Heslot, A. Quantum mechanics as a classical theory. Phys. Rev. D 1985, 31, 1341. [CrossRef]

38. Cirelli, R.; Mania, A.; Pizzocchero, L. Quantum mechanics as an infinite-dimensional Hamiltonian system with uncertainty structure: Part I. J. Math. Phys. 1990, 31, 2891-2897. [CrossRef]

39. Cirelli, R.; Mania, A.; Pizzocchero, L. Quantum mechanics as an infinite-dimensional Hamiltonian system with uncertainty structure: Part II. J. Math. Phys. 1990, 31, 2898-2903. [CrossRef]

40. Ashtekar, A.; Schilling, T.A. Geometrical formulation of quantum mechanics. In On Einstein's Path: Essays in Honor of Engelbert Schücking; Harvey, A., Ed.; Springer: New York, NY, USA, 1999; pp. 23-65.

41. Wallstrom, T.C. Inequivalence between the Schrödinger equation and the Madelung hydrodynamic equations. Phys. Rev. A 1994, 49, 1613. [CrossRef]

42. Dirac, P.A.M. The Hamiltonian form of field dynamics. Can. J. Math. 1951, 3, 1-23. [CrossRef]

43. Dirac, P.A.M. Lectures on Quantum Mechanics, 2nd ed.; Courier Corporation: Chelmsford, MA, USA, 2013.

44. Hojman, S.A.; Kuchař, K.; Teitelboim, C. Geometrodynamics regained. Ann. Phys. 1976, 96, 88-135. [CrossRef]

45. Kuchař, K. A Bubble-Time Canonical Formalism for Geometrodynamics. J. Math. Phys. 1972, 13, 768-781. [CrossRef]

46. Teitelboim, C. How commutators of constraints reflect the spacetime structure. Ann. Phys. 1973, 79, 542-557. [CrossRef]

47. Weitzman, C.T. The Hamiltonian Structure of Spacetime. Princeton University, Ph.D. Thesis, Princeton, NJ, USA, 1973.

48. Weiss, P. On the Hamilton-Jacobi theory and quantization of a dynamical continuum. Proc. R. Soc. Lond. A 1938, 169. [CrossRef]

49. Tomonaga, S. On a relativistically invariant formulation of the quantum theory of wave fields. Prog. Theor. Exp. Phys. 1946, 1, 27-42. [CrossRef]

50. Dirac, P.A.M. Relativistic quantum mechanics. Proc. R. Soc. Lond. A 1932, 136. [CrossRef]

51. Schwinger, J. Quantum electrodynamics. I. A covariant formulation. Phys. Rev. 1948, 74, 1439. [CrossRef]

52. Wald, R.M. Quantum Field Theory in Curved Spacetime and Black Hole Thermodynamics; University of Chicago Press: Chicago, IL, USA, 1994.

53. Stapp, H.P. The copenhagen interpretation. Am. J. Phys. 1972, 40, 1098-1116. [CrossRef] 
54. Leifer, M.S. Is the quantum state real? An extended review of $\psi$-ontology theorems. arXiv 2014, arXiv:1409.1570.

55. Everett, H., III. "Relative state" formulation of quantum mechanics. Rev. Mod. Phys. 1957, 29, 454. [CrossRef]

56. Bartolomeo, D.; Caticha, A. Trading drift and fluctuations in entropic dynamics: Quantum dynamics as an emergent universality class. In Proceedings of the Emergent Quantum Mechanics 2015, Vienna, Austria, 23-25 October 2015; Volume 701.

57. Dirac, P.A.M. The theory of gravitation in Hamiltonian form. Proc. R. Soc. Lond. A 1958, 246, 333-343.

58. Arnowitt, R.; Deser, S.; Misner, C.W. Canonical variables for general relativity. Phys. Rev. 1960, 117, 1595. [CrossRef]

59. Arnowitt, R.; Deser, S.; Misner, C.W. Republication of: The dynamics of general relativity. Gen. Relativ. Gravit. 2008, 40, 1997-2027. [CrossRef]

60. Gourgoulhon, E. 3+ 1 formalism and bases of numerical relativity. arXiv 2007, arXiv: Gr-qc/0703035.

61. Misner, C.W.; Thorne, K.S.; Wheeler, J.A. Gravitation; Macmillan: New York, NY, USA, 1973.

62. Ferraris, M.; Kijowski, J. General relativity is a gauge type theory. Lett. Math. Phys. 1981, 5, 127-135. [CrossRef]

63. Ashtekar, A. New variables for classical and quantum gravity. Phys. Rev. Lett. 1986, 57, 2244. [CrossRef]

64. Schutz, B.F. Geometrical Methods of Mathematical Physics; Cambridge University Press: Cambridge, UK, 1980.

65. Carroll, S.M. Spacetime and Geometry: An Introduction to General Relativity; Addison Wesley: Boston, MA, USA, 2004.

66. Kibble, T.W.B.; Randjbar-Daemi, S. Non-linear coupling of quantum theory and classical gravity. J. Phys. A 1980, 13, 141. [CrossRef]

67. Unruh, W.G. Steps towards a quantum theory of gravity. In Quantum Theory of Gravity. Essays in Honor of the 60th Birthday of Bryce S. DeWitt; DeWitt, B.S., Ed.; Adam Hilger Ltd.: Bristol, UK, 1984; pp. 234-242.

68. Bahrami, M.; Großardt, A.; Donadi, S.; Bassi, A. The Schrödinger-Newton equation and its foundations. New J. Phys. 2014, 16, 115007. [CrossRef]

69. Eppley, K.; Hannah, E. The necessity of quantizing the gravitational field. Found. Phys. 1977, 7, 51-68. [CrossRef]

70. Page, D.N.; Geilker, C.D. Indirect evidence for quantum gravity. Phys. Rev. Lett. 1981, 47, 979. [CrossRef]

71. Duff, M.J. Quantum Gravity 2: A Second Oxford Symposium; Isham, C.J., Penrose, R., Sciama, D.W., Eds.; Clarendon Press: Oxford, UK, 1981.

72. Kibble, T.W.B. Relativistic models of nonlinear quantum mechanics. Commun. Math. Phys. 1978, 64, 73-82. [CrossRef]

(C) 2020 by the authors. Licensee MDPI, Basel, Switzerland. This article is an open access article distributed under the terms and conditions of the Creative Commons Attribution (CC BY) license (http://creativecommons.org/licenses/by/4.0/). 